\title{
Dominica: Statistical Appendix
}

This Statistical Appendix for Dominica was prepared by a staff team of the International Monetary Fund as background documentation for the periodic consultation with the member country. It is based on the information available at the time it was completed on September 30, 2005. The views expressed in this document are those of the staff team and do not necessarily reflect the views of the government of Dominica or the Executive Board of the IMF.

The policy of publication of staff reports and other documents by the IMF allows for the deletion of market-sensitive information.

To assist the IMF in evaluating the publication policy, reader comments are invited and may be sent by e-mail to publicationpolicy@imf.org.

Copies of this report are available to the public from

International Monetary Fund $\bullet$ Publication Services

700 19th Street, N.W. • Washington, D.C. 20431

Telephone: (202) 6237430 • Telefax: (202) 6237201

E-mail: publications@imf.org • Internet: http://www.imf.org

Price: $\$ 15.00$ a copy

\section{International Monetary Fund Washington, D.C.}





\section{INTERNATIONAL MONETARY FUND}

\section{DOMINICA}

\section{Statistical Appendix \\ Prepared by a staff team consisting of Patrick Njoroge, Pawel Dyczewski, Tobias Rasmussen (all WHD), and Pedro Rodriguez (PDR) \\ Approved by Western Hemisphere Department}

September 30, 2005

Contents

Page

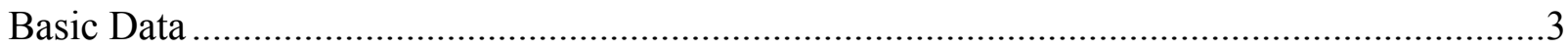

Tables

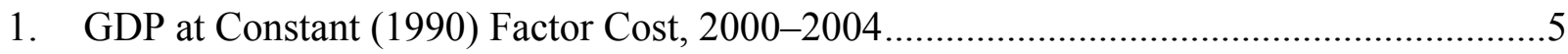

2. GDP at Current Factor Cost, 2000-2004 ......................................................................

3. Aggregate Domestic Expenditure at Current Market Prices, 2000-2004 ……….................7

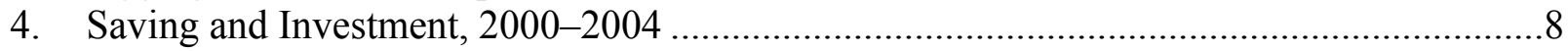

5. Selected Agricultural Production, 2000-2004 ...................................................................

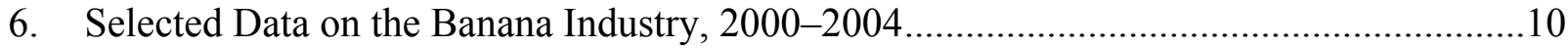

7. Selected Industrial Production, 2000-2004 ...............................................................11

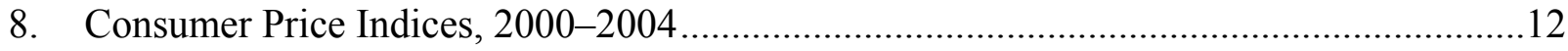

9. Petroleum Imports and Retail Prices, 2000-2004 …….................................................13

10. Summary Accounts of the Consolidated Public Sector, 1999/00-2003/04 .........................14

11. Summary Accounts of the Central Government, 2000/01-2004/05 .................................17

12. Central Government Revenue and Grants, 2000/01-2004/05 …………………...............19

13. Central Government Expenditure, 2000/01-2004/05 ........................................................21

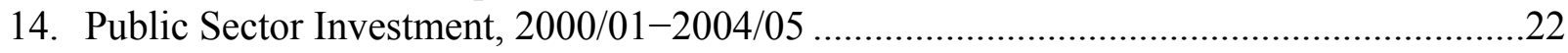

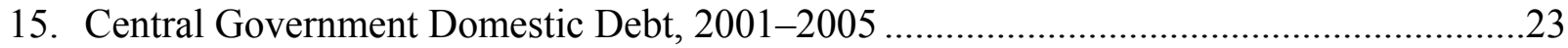

16. Consolidated Accounts of the Public Enterprises, 1999/00-2003/04 ................................24

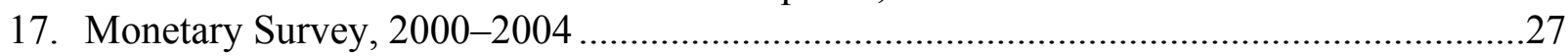

18. Balance Sheet of the Monetary Authorities, 2000-2004 ………….................................28

19. Consolidated Balance Sheet of Commercial Banks, 2000-2004 ……...............................29

20. Sectoral Distribution of Loans and Advances of

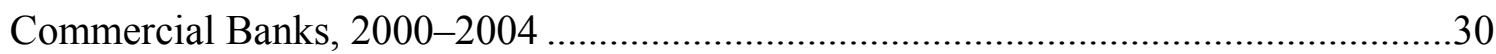

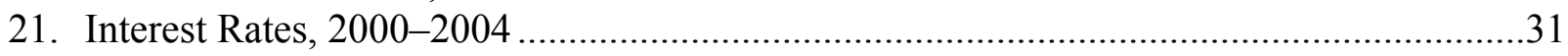

22. Financial Accounts of the Dominica Agricultural and

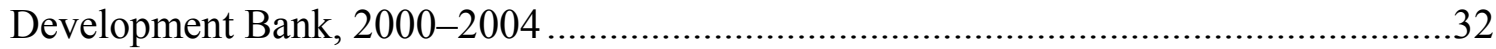

23. Consolidated Balance Sheet of the Major Credit Unions, 2000-2004 ...............................33 


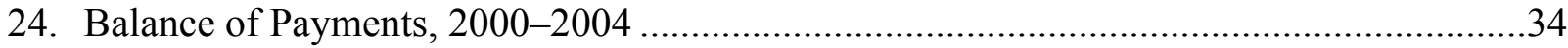

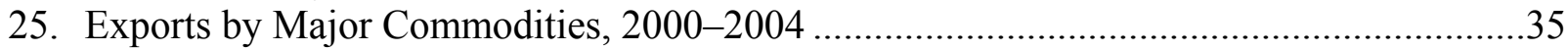

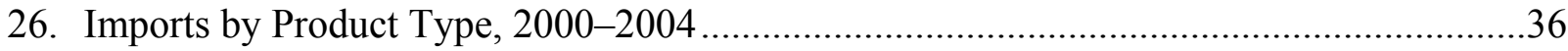

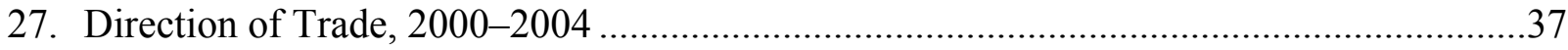

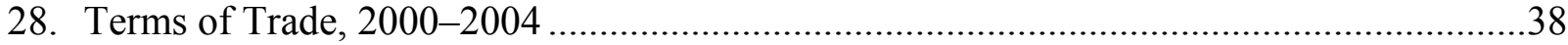

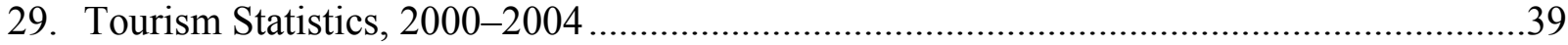

30. Effective Exchange Rate Indices, 2000-2004 .............................................................40

31. External Public and Publicly Guaranteed Debt by Creditor, 2000-2004 ........................41 


\section{Dominica: Basic Data}

I. Social and Demographic Indicators

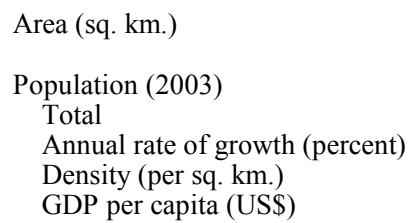

Population characteristics (most recent year) Life expectancy at birth (years) Infant mortality (per thousand live births)

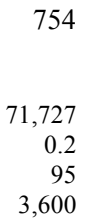

Nutrition (1994)

Calorie intake (percent of requirement)

Protein intake (grams/day/person)

Education (1992)

Gross enrollment rate, in percent

Primary education

71

GDP (2004)

Millions of E.C. dollars $\quad 731.7$

Millions of U.S. dollars 271.0

II. Economic Indicators, 2000-2004

Prel.

(In percent of GDP)

\section{Origin of GDP}

Agriculture and mining

Manufacturing and construction

Services

$\begin{array}{lllll}18.9 & 18.5 & 19.3 & 18.3 & 18.3 \\ 17.2 & 16.2 & 15.0 & 15.6 & 16.0 \\ 58.4 & 60.1 & 60.4 & 60.8 & 60.3\end{array}$

(Annual percentage changes, unless otherwise indicated)
National accounts and prices

Real GDP

GDP deflator (factor cost)

Nominal GDP at market prices

Consumer price index (period average)

Consumer price index (end of period)

$\begin{array}{rr}1.4 & -4.2 \\ 0.6 & 1.2 \\ 1.0 & -2.7 \\ \ldots & 1.6 \\ 1.1 & 1.1\end{array}$

1.1

(In percent of GDP)
Gross domestic investment

$$
\text { Of which }
$$

Public investment

Gross national savings

External savings

Private consumption

Public consumption

Public finances 1/

Central government

Total revenues and grants

Total expenditure

Of which

Interest payments

Capital expenditure and net lending

Current account balance

Overall balance

Consolidated public sector

Current account balance

Fixed investment

Overall balance
27.7

18.6

12.0

15.7

64.0

22.6

37.6

48.5

5.0

16.6

$-4.1$

$-10.9$

$-2.2$

20.3

$-10.2$
$-4.7$

$-0.4$

$-3.7$

0.1

0.4

$\begin{array}{ll}0.0 & 3.5 \\ 1.7 & 1.2 \\ 2.4 & 5.0 \\ 1.6 & 2.4 \\ 2.9 & 0.8\end{array}$


Dominica: Basic Data

\begin{tabular}{llllll}
\hline & 2000 & 2001 & 2002 & 2003 & 2004 \\
\hline
\end{tabular}

(12-month percentage changes, unless otherwise indicated)

\section{Money and credit}

\section{Broad money}

Money

Quasi money

Net domestic assets of the banking system 2/

Of which

Credit to the nonfinancial public sector (net) 2/

Credit to the nonfinancial private sector $2 /$

Representative interest rate (in percent) 3 /

$\begin{array}{rr}0.6 & 7.4 \\ -15.4 & 1.4 \\ 5.3 & 8.9 \\ 13.4 & 0.9 \\ & \\ 5.7 & 5.6 \\ 7.3 & -3.1 \\ 11.7 & 11.0\end{array}$

8.5
16.5
6.8
-10.8

1.0

5.9

$0.9 \quad 6.6$

$\begin{array}{lll}-5.4 & -4.3 & -5.1\end{array}$

(In millions of U.S. dollars, unless otherwise indicated)

Balance of payments

Current account

Merchandise trade balance

Exports

Imports

Services, income, and transfers (net)

Of which: interest paid on public debt

Capital and financial account

Public sector

Private sector (including errors and omissions)

Change in net international reserves (- increase) 5/

Merchandise exports (in percent of GDP)

Merchandise imports (in percent of GDP)

Current account (in percent of GDP)

Merchandise exports (in US\$, annual percentage change)

Merchandise imports (in US\$, annual percentage change)

Terms of trade (annual percentage change)

Real effective exchange rate (12-month percentage change) 4 /

\begin{tabular}{|c|c|c|c|c|}
\hline-53.1 & -48.9 & -34.7 & -33.6 & -46.7 \\
\hline-75.6 & -71.3 & -58.7 & -70.8 & -84.6 \\
\hline 54.7 & 44.4 & 43.6 & 41.0 & 42.2 \\
\hline 130.4 & 115.7 & 102.4 & 111.8 & 126.9 \\
\hline 22.6 & 22.4 & 24.0 & 37.3 & 38.0 \\
\hline-6.2 & -7.7 & -8.8 & -8.0 & -8.6 \\
\hline 50.5 & 50.3 & 46.9 & 35.0 & 20.9 \\
\hline 31.6 & 35.4 & 43.4 & 24.8 & 24.0 \\
\hline 18.9 & 14.9 & 3.4 & 10.2 & -3.1 \\
\hline 2.5 & -1.5 & -12.1 & -1.4 & 10.3 \\
\hline 20.4 & 17.0 & 17.3 & 15.9 & 15.6 \\
\hline 48.5 & 44.2 & 40.6 & 43.3 & 46.8 \\
\hline-19.7 & -18.7 & -13.8 & -13.0 & -17.2 \\
\hline-2.3 & -18.9 & -1.8 & -6.0 & 3.0 \\
\hline 7.3 & -11.3 & -11.5 & 9.3 & 13.5 \\
\hline-16.3 & 5.8 & 4.9 & 6.1 & $\ldots$ \\
\hline 4.8 & 3.7 & -6.3 & -6.7 & -7.0 \\
\hline 29.0 & 30.4 & 43.6 & 44.0 & 33.6 \\
\hline 24.2 & 35.6 & 71.8 & 107.0 & 123.6 \\
\hline 56.4 & 67.5 & 79.9 & 84.4 & 81.2 \\
\hline 7.4 & 10.9 & 11.8 & 19.5 & 20.8 \\
\hline
\end{tabular}

International reserve position and

external debt (as of December 31)

Net official reserves 5/

Net reserves of the banking system

External public and publicly

guaranteed debt (in percent of GDP)

Debt service ratio (in percent of exports of

goods and services)

$-1.3$

$-2.3 \quad 4.3$

$\begin{array}{lll}11.0 & 11.8 & 9.8\end{array}$

IMF data (as of August 31, 2005)

Membership status: joined December 12, 1978

Intervention currency and rate

Quota

Fund holdings of EC dollars (Dominica)

(As percent of quota)

Outstanding purchases and loans

SDR department

Net cumulative allocation

Holdings

Article VIII

U.S. dollar at EC\$2.70 per U.S. dollar

SDR 8.20 million

SDR 11.16 million

136.15 percent

SDR 7.18 million

SDR 0.59 million SDR 0.01 million

Sources: Dominican authorities; and Fund staff estimates.

1/ These data are presented on a fiscal year (July-June) basis. Figures shown for a given calendar year relate to the fiscal year beginning on July 1 of that year.

2/ Percentage change in terms of broad money stock 12 months earlier.

3/ Weighted average lending rate.

4/ A negative sign means a depreciation.

5/ Imputed reserves at the Eastern Caribbean Central Bank. 
Table 1. Dominica: GDP at Constant (1990) Factor Cost, 2000-2004

\begin{tabular}{|c|c|c|c|c|c|}
\hline & 2000 & 2001 & 2002 & 2003 & $\begin{array}{l}\text { Prel. } \\
2004 \\
\end{array}$ \\
\hline \multicolumn{6}{|c|}{ (In millions of 1990 Eastern Caribbean dollars) } \\
\hline GDP at 1990 factor cost & 456.7 & 437.5 & 417.1 & 417.1 & 431.5 \\
\hline Agriculture & 82.0 & 76.6 & 76.1 & 72.4 & 74.6 \\
\hline Crops & 62.8 & 57.2 & 56.5 & 52.6 & 54.6 \\
\hline Livestock & 7.4 & 7.5 & 7.6 & 7.6 & 7.6 \\
\hline Forestry & 3.4 & 3.4 & 3.5 & 3.5 & 3.5 \\
\hline Fishing & 8.3 & 8.5 & 8.6 & 8.7 & 8.9 \\
\hline Manufacturing & 3.7 & 3.6 & 2.8 & 2.9 & 3.1 \\
\hline Mining and quarrying & 31.6 & 27.2 & 27.1 & 28.3 & 29.6 \\
\hline Electricity and water & 19.7 & 20.3 & 20.4 & 20.1 & 21.1 \\
\hline Construction & 37.0 & 36.1 & 27.9 & 29.1 & 31.2 \\
\hline Wholesale and retail trade & 58.5 & 58.0 & 55.0 & 56.0 & 58.2 \\
\hline Hotels and restaurants & 11.7 & 10.8 & 10.2 & 10.7 & 11.9 \\
\hline Transport & 44.7 & 42.2 & 36.3 & 39.7 & 45.9 \\
\hline Road transport & 25.5 & 23.7 & 19.3 & 21.8 & 26.4 \\
\hline Sea transport & 15.4 & 14.8 & 13.6 & 14.4 & 15.8 \\
\hline Air transport & 3.9 & 3.7 & 3.4 & 3.5 & 3.7 \\
\hline Communications & 53.2 & 49.7 & 45.0 & 41.9 & 44.4 \\
\hline Banks and insurance & 58.5 & 56.5 & 57.9 & 59.2 & 60.3 \\
\hline Real estate and housing & 15.7 & 15.8 & 16.0 & 16.1 & 16.4 \\
\hline Government services & 82.0 & 85.8 & 88.9 & 87.9 & 83.7 \\
\hline Other services & 6.0 & 6.1 & 6.2 & 6.3 & 6.4 \\
\hline Less imputed service charge & 47.4 & 51.4 & 52.8 & 53.4 & 55.4 \\
\hline \multicolumn{6}{|c|}{ (In percentage change) } \\
\hline GDP at 1990 factor cost & 1.4 & -4.2 & -4.7 & $\mathbf{0 . 0}$ & 3.5 \\
\hline Agriculture & -1.1 & -6.6 & -0.6 & -4.9 & 3.0 \\
\hline Crops & -1.4 & -8.9 & -1.2 & -6.9 & 3.8 \\
\hline Livestock & 1.0 & 1.0 & 0.9 & 0.7 & 0.1 \\
\hline Forestry & 0.9 & 1.0 & 0.8 & 0.0 & 0.3 \\
\hline Fishing & -1.2 & 1.4 & 1.4 & 1.2 & 2.0 \\
\hline Manufacturing & -87.3 & -2.2 & -22.9 & 4.4 & 7.1 \\
\hline Mining and quarrying & 806.3 & -14.0 & -0.3 & 4.3 & 4.7 \\
\hline Electricity and water & 2.5 & 3.3 & 0.6 & -1.8 & 5.3 \\
\hline Construction & 6.1 & -2.2 & -22.9 & 4.6 & 7.1 \\
\hline Wholesale and retail trade & 2.5 & -0.7 & -5.2 & 1.7 & 4.0 \\
\hline Hotels and restaurants & 3.9 & -7.4 & -5.5 & 4.7 & 11.0 \\
\hline Transport & -1.0 & -5.5 & -14.1 & 9.5 & 15.6 \\
\hline Road transport & 4.9 & -6.8 & -18.7 & 13.1 & 21.1 \\
\hline Sea transport & -9.3 & -3.6 & -8.4 & 6.4 & 9.5 \\
\hline Air transport & -1.5 & -4.4 & -7.6 & 1.5 & 6.3 \\
\hline Communications & -4.4 & -6.5 & -9.5 & -6.9 & 6.0 \\
\hline Banks and insurance & 0.3 & -3.3 & 2.4 & 2.2 & 2.0 \\
\hline Real estate and housing & 1.0 & 1.0 & 0.9 & 0.7 & 2.0 \\
\hline Government services & 4.4 & 4.6 & 3.7 & -1.2 & -4.8 \\
\hline Other services & -0.5 & 2.7 & 1.6 & 1.2 & 2.0 \\
\hline Less imputed service charge & 2.0 & 8.5 & 2.7 & 1.3 & 3.6 \\
\hline
\end{tabular}

Source: Central Statistical Office, Ministry of Finance. 
Table 2. Dominica: GDP at Current Factor Cost, 2000-2004

\begin{tabular}{|c|c|c|c|c|c|}
\hline & 2000 & 2001 & 2002 & 2003 & $\begin{array}{r}\text { Prel. } \\
2004 \\
\end{array}$ \\
\hline \multicolumn{6}{|c|}{ (In millions of Eastern Caribbean dollars) } \\
\hline GDP at current factor cost & 621.6 & 602.8 & 572.3 & 582.1 & 609.5 \\
\hline Agriculture & 112.5 & 106.4 & 106.3 & 102.3 & 107.1 \\
\hline Crops & 90.1 & 83.7 & 83.4 & 78.8 & 83.0 \\
\hline Livestock & 8.3 & 8.4 & 8.4 & 8.6 & 8.8 \\
\hline Forestry & 3.6 & 3.7 & 3.7 & 3.7 & 3.8 \\
\hline Fishing & 10.6 & 10.7 & 10.9 & 11.2 & 11.5 \\
\hline Manufacturing & 5.2 & 5.2 & 4.1 & 4.3 & 4.7 \\
\hline Mining and quarrying & 54.4 & 45.1 & 45.1 & 47.7 & 50.7 \\
\hline Electricity and water & 33.9 & 31.3 & 30.7 & 30.6 & 32.7 \\
\hline Construction & 52.2 & 52.4 & 40.6 & 43.1 & 46.8 \\
\hline Wholesale and retail trade & 72.6 & 73.4 & 69.7 & 72.0 & 76.0 \\
\hline Hotels and restaurants & 17.0 & 16.0 & 15.1 & 16.1 & 18.1 \\
\hline Transport & 57.7 & 53.0 & 45.1 & 50.1 & 58.6 \\
\hline Road transport & 29.8 & 27.7 & 22.5 & 25.9 & 31.8 \\
\hline Sea transport & 23.1 & 20.7 & 18.6 & 20.1 & 22.3 \\
\hline Air transport & 4.9 & 4.5 & 4.0 & 4.1 & 4.4 \\
\hline Communications & 47.8 & 44.3 & 34.4 & 32.5 & 35.0 \\
\hline Banks and insurance & 70.1 & 70.1 & 67.4 & 69.8 & 72.3 \\
\hline Real estate and housing & 20.9 & 21.3 & 21.8 & 22.3 & 23.1 \\
\hline Government services & 121.7 & 128.4 & 133.3 & 133.7 & 129.2 \\
\hline Other services & 9.5 & 9.9 & 10.1 & 10.4 & 10.8 \\
\hline Less imputed service charge & 54.0 & 54.1 & 51.4 & 52.8 & 55.5 \\
\hline \multicolumn{6}{|c|}{ (In percent of GDP at current factor cost) } \\
\hline GDP at current factor cost & 100.0 & 100.0 & 100.0 & 100.0 & 100.0 \\
\hline Agriculture & 18.1 & 17.6 & 18.6 & 17.6 & 17.6 \\
\hline Crops & 14.5 & 13.9 & 14.6 & 13.5 & 13.6 \\
\hline Livestock & 1.3 & 1.4 & 1.5 & 1.5 & 1.4 \\
\hline Forestry & 0.6 & 0.6 & 0.6 & 0.6 & 0.6 \\
\hline Fishing & 1.7 & 1.8 & 1.9 & 1.9 & 1.9 \\
\hline Manufacturing & 0.8 & 0.9 & 0.7 & 0.7 & 0.8 \\
\hline Mining and quarrying & 8.8 & 7.5 & 7.9 & 8.2 & 8.3 \\
\hline Electricity and water & 5.5 & 5.2 & 5.4 & 5.3 & 5.4 \\
\hline Construction & 8.4 & 8.7 & 7.1 & 7.4 & 7.7 \\
\hline Wholesale and retail trade & 11.7 & 12.2 & 12.2 & 12.4 & 12.5 \\
\hline Hotels and restaurants & 2.7 & 2.7 & 2.6 & 2.8 & 3.0 \\
\hline Transport & 9.3 & 8.8 & 7.9 & 8.6 & 9.6 \\
\hline Road transport & 4.8 & 4.6 & 3.9 & 4.4 & 5.2 \\
\hline Sea transport & 3.7 & 3.4 & 3.2 & 3.4 & 3.7 \\
\hline Air transport & 0.8 & 0.8 & 0.7 & 0.7 & 0.7 \\
\hline Communications & 7.7 & 7.4 & 6.0 & 5.6 & 5.7 \\
\hline Banks and insurance & 11.3 & 11.6 & 11.8 & 12.0 & 11.9 \\
\hline Real estate and housing & 3.4 & 3.5 & 3.8 & 3.8 & 3.8 \\
\hline Government services & 19.6 & 21.3 & 23.3 & 23.0 & 21.2 \\
\hline Other services & 1.5 & 1.6 & 1.8 & 1.8 & 1.8 \\
\hline Less imputed service charge & 8.7 & 9.0 & 9.0 & 9.1 & 9.1 \\
\hline
\end{tabular}

Source: Central Statistical Office, Ministry of Finance. 
Table 3. Dominica: Aggregate Domestic Expenditure at Current Market Prices, 2000-2004

\begin{tabular}{|c|c|c|c|c|c|}
\hline & 2000 & 2001 & 2002 & 2003 & $\begin{array}{l}\text { Prel. } \\
2004 \\
\end{array}$ \\
\hline \multicolumn{6}{|c|}{ (In millions of Eastern Caribbean dollars) } \\
\hline Gross domestic expenditure & 830.5 & 830.3 & 769.0 & 799.9 & 852.3 \\
\hline Consumption & 629.5 & 651.1 & 626.6 & 622.5 & 641.0 \\
\hline Private & 465.1 & 490.7 & 479.2 & 487.4 & 501.3 \\
\hline Public & 164.4 & 160.4 & 147.3 & 135.1 & 139.7 \\
\hline Gross domestic investment & 201.1 & 179.2 & 142.4 & 177.3 & 211.3 \\
\hline Private & 66.3 & 72.9 & 91.4 & 111.7 & 125.2 \\
\hline Public & 134.8 & 106.3 & 51.0 & 65.6 & 86.1 \\
\hline Exports of goods and nonfactor services & -104.2 & -123.4 & -88.5 & -102.8 & -120.5 \\
\hline Exports & 390.1 & 324.1 & 333.0 & 319.5 & 347.2 \\
\hline Imports & -494.3 & -447.5 & -421.4 & -422.3 & -467.7 \\
\hline GDP at current market prices & 726.4 & 706.8 & 680.5 & 697.1 & 731.7 \\
\hline \multicolumn{6}{|c|}{ (In percent of GDP) } \\
\hline Gross domestic expenditure & 114.3 & 117.5 & 113.0 & 114.7 & 116.5 \\
\hline Consumption & 86.7 & 92.1 & 92.1 & 89.3 & 87.6 \\
\hline Private & 64.0 & 69.4 & 70.4 & 69.9 & 68.5 \\
\hline Public & 22.6 & 22.7 & 21.6 & 19.4 & 19.1 \\
\hline Gross domestic investment & 27.7 & 25.4 & 20.9 & 25.4 & 28.9 \\
\hline Private & 9.1 & 10.3 & 13.4 & 16.0 & 17.1 \\
\hline Public & 18.6 & 15.0 & 7.5 & 9.4 & 11.8 \\
\hline Exports of goods and nonfactor services & -14.3 & -17.5 & -13.0 & -14.7 & -16.5 \\
\hline Exports & 53.7 & 45.9 & 48.9 & 45.8 & 47.5 \\
\hline Imports & -68.1 & -63.3 & -61.9 & -60.6 & -63.9 \\
\hline GDP at current market prices & 100.0 & 100.0 & 100.0 & 100.0 & 100.0 \\
\hline
\end{tabular}

Sources: Ministry of Finance; and Fund staff estimates. 
Table 4. Dominica: Saving and Investment, 2000-2004

\begin{tabular}{|c|c|c|c|c|c|}
\hline & 2000 & 2001 & 2002 & 2003 & $\begin{array}{l}\text { Prel. } \\
2004\end{array}$ \\
\hline \multicolumn{6}{|c|}{ (In millions of Eastern Caribbean dollars) } \\
\hline Gross national saving & 87.2 & 95.8 & 104.0 & 137.4 & 157.7 \\
\hline Private saving & 60.1 & 70.6 & 103.9 & 96.0 & 79.0 \\
\hline Public saving & 27.1 & 25.2 & 0.0 & 41.4 & 78.7 \\
\hline Foreign saving & 113.9 & 83.4 & 38.4 & 39.9 & 53.6 \\
\hline Gross domestic investment & 201.1 & 179.2 & 142.4 & 177.3 & 211.3 \\
\hline Private investment & 66.3 & 72.9 & 91.4 & 111.7 & 125.2 \\
\hline Public investment & 134.8 & 106.3 & 51.0 & 65.6 & 86.1 \\
\hline \multicolumn{6}{|c|}{ (In percent of GDP) } \\
\hline Gross national saving & 12.0 & 13.6 & 15.3 & 19.7 & 21.6 \\
\hline Private saving & 8.3 & 10.0 & 15.3 & 13.8 & 10.8 \\
\hline Public saving & 3.7 & 3.6 & 0.0 & 5.9 & 10.8 \\
\hline Foreign saving & 15.7 & 11.8 & 5.6 & 5.7 & 7.3 \\
\hline Gross domestic investment & 27.7 & 25.4 & 20.9 & 25.4 & 28.9 \\
\hline Private investment & 9.1 & 10.3 & 13.4 & 16.0 & 17.1 \\
\hline Public investment & 18.6 & 15.0 & 7.5 & 9.4 & 11.8 \\
\hline
\end{tabular}

Sources: Ministry of Finance; and Fund staff estimates. 
Table 5. Dominica: Selected Agricultural Production, 2000-2004

(In metric tons)

\begin{tabular}{|c|c|c|c|c|c|}
\hline & 2000 & 2001 & 2002 & 2003 & $\begin{array}{l}\text { Prel. } \\
2004 \\
\end{array}$ \\
\hline Avocado pears & 861 & 562 & 559 & 698 & 770 \\
\hline Bananas & 60,896 & 25,048 & 24,645 & 16,058 & 19,344 \\
\hline Coconuts & 42 & 10,675 & 9,737 & 9,669 & 9,656 \\
\hline Bay oil & 27 & 23 & 30 & 27 & 35 \\
\hline Beans & 89 & 89 & 88 & 90 & 90 \\
\hline Breadfruit & 280 & 283 & 268 & 274 & 271 \\
\hline Cabbage & 909 & 912 & 903 & 910 & 910 \\
\hline Carrot & 523 & 525 & 522 & 525 & 525 \\
\hline Cassava & 92 & 97 & 97 & 88 & 90 \\
\hline Christophene & 239 & 233 & 222 & 226 & 226 \\
\hline Cinnamon & 15 & 15 & 16 & 18 & 17 \\
\hline Cocoa beans & 74 & 81 & 80 & 76 & 74 \\
\hline Coffee & 207 & 186 & 187 & 185 & 181 \\
\hline Cucumber & 1,032 & 1,029 & 1,004 & 956 & 922 \\
\hline Cut flowers & 48 & 54 & 46 & 47 & 45 \\
\hline Dasheen & 11,723 & 11,559 & 11,563 & 11,378 & 11,655 \\
\hline Ginger & 66 & 82 & 84 & 79 & 82 \\
\hline Grapefruit & 18,172 & 18,301 & 18,620 & 16,899 & 16,819 \\
\hline Irish potatoes & 154 & 154 & 154 & 159 & 149 \\
\hline Lettuce & 153 & 153 & 151 & 152 & 151 \\
\hline Limes & 1,353 & 1,285 & 1,250 & 1,128 & 1,087 \\
\hline Mandarines & 726 & 39 & 43 & 484 & 476 \\
\hline Mango & 1,716 & 1,682 & 1,628 & 1,654 & 1,627 \\
\hline Melongene & 67 & 59 & 33 & 26 & 36 \\
\hline Nutmet & 2 & 2 & 2 & 2 & 1 \\
\hline Okra & 3 & 3 & 3 & 2 & 2 \\
\hline Oranges & 3,544 & 3,594 & 3,517 & 3,528 & 3,499 \\
\hline Papaya & 113 & 98 & 83 & 82 & 70 \\
\hline Passionfruit & 471 & 456 & 448 & 443 & 446 \\
\hline Peppers (hot) & 124 & 116 & 136 & 218 & 229 \\
\hline Peppers (sweet) & $\ldots$ & $\ldots$ & $\ldots$ & 163 & 156 \\
\hline Pineapple & $\ldots$ & $\ldots$ & $\ldots$ & 338 & 361 \\
\hline Plantain & 8,043 & 7,646 & 7,556 & 7,644 & 7,808 \\
\hline Pumpkin & 813 & 765 & 715 & 792 & 726 \\
\hline Sweet corn & $\ldots$ & $\ldots$ & $\ldots$ & 3 & 3 \\
\hline Sweet potato & 1,691 & 1,709 & 1,643 & 1,681 & 1,678 \\
\hline Tannia & 3,400 & 3,295 & 3,267 & 3,339 & 3,412 \\
\hline Tomato & 203 & 203 & 200 & 206 & 205 \\
\hline Water melon & 250 & 262 & 255 & 253 & 251 \\
\hline Yam & 6,862 & 6,835 & 6,707 & 6,952 & 6,948 \\
\hline Total & 124,981 & 98,112 & 96,461 & 87,448 & 91,033 \\
\hline
\end{tabular}

Source: Central Statistical Office, Ministry of Finance. 
Table 6. Dominica: Selected Data on the Banana Industry, 2000-2004

\begin{tabular}{|c|c|c|c|c|c|}
\hline & 2000 & 2001 & 2002 & 2003 & $\begin{array}{l}\text { Prel. } \\
2004\end{array}$ \\
\hline \multicolumn{6}{|c|}{ (In metric tons; unless otherwise indicated) } \\
\hline Gross banana purchases by the DBMC/DBPL & 28,241 & 18,231 & 18,042 & 11,212 & 13,875 \\
\hline Less rejects $1 /$ & 1,009 & 616 & 854 & 649 & 713 \\
\hline Net purchases by DBMC/DBPL & 27,232 & 17,615 & 17,188 & 10,563 & 13,162 \\
\hline DBMC/DBPL exports 2/ & 27,232 & 17,615 & 17,188 & 10,558 & 12,976 \\
\hline Left back & 0 & 0 & 0 & 5 & 186 \\
\hline $\begin{array}{l}\text { DBMC/DBPL export receipts } \\
\text { (In thousands of E.C. dollars) }\end{array}$ & 29,800 & 20,202 & 20,172 & 13,530 & 17,214 \\
\hline $\begin{array}{l}\text { Average retail price in the United Kingdom } \\
\text { (In pence per kilogram) }\end{array}$ & 99.0 & 106.0 & 103.0 & 92.0 & 86.0 \\
\hline $\begin{array}{l}\text { Free-on-truck (FOT) price } 3 / \\
\text { (In pounds sterling per metric ton) }\end{array}$ & 436.0 & 465.5 & $\ldots$ & $\ldots$ & ... \\
\hline Number of active growers 4/ & 2,410 & 2,125 & 1,488 & 1,149 & 1,021 \\
\hline Number of certified growers & 1,656 & 1,231 & 978 & 911 & 925 \\
\hline Quality rating (percent of export volume) 5/ & 91 & 90 & 79 & 89 & 87 \\
\hline \multicolumn{6}{|c|}{ (In Eastern Caribbean cents per pound) } \\
\hline FOT price & 81.0 & 82.1 & $\ldots$ & $\ldots$ & $\ldots$ \\
\hline DBMC/DBPL export unit value (f.o.b.) & 49.6 & 52.0 & 52.0 & 55.0 & 57.0 \\
\hline Average gross price to growers & 60.1 & 48.7 & 47.0 & 50.0 & 51.0 \\
\hline Average net price to growers $6 /$ & 29.8 & 21.7 & 24.0 & 23.0 & 26.0 \\
\hline \multicolumn{6}{|l|}{ Memoranda items: } \\
\hline \multicolumn{6}{|l|}{ Export market prices (U.S. dollars per metric tonne) } \\
\hline Latin American bananas, FOB U.S. ports & 422.3 & 584.7 & 527.6 & 375.2 & 524.8 \\
\hline Latin American bananas, c.i.f. Hamburg & 712.4 & 777.2 & 759.4 & 790.4 & 892.0 \\
\hline $\begin{array}{l}\text { Average gross price to growers as } \\
\text { percent of DBMC/DBPL's export unit value }\end{array}$ & 121.1 & 93.6 & 90.4 & 90.9 & 89.5 \\
\hline
\end{tabular}

Sources: Dominica Banana Marketing Corporation (DBMC)/Dominica Banana Producers Ltd. (DBPL); Central Statistical Office, Ministry of Finance; and World Bank database.

1/ Bananas purchased by the not meeting quality standards.

2/ The European market (mainly the United Kingdom) accounts for about 95 percent of banana exports.

3/ The FOT price is the price at the point of delivery for shipment to EU markets.

4/ Total number of growers which contributed to production at any time during given year.

5/ Percentage Units within Specification (PUWS), is the quality measure established by the Windward

Islands Banana Development and Exporting Company (WIBDECO).

6/ Net of DBMC's deductions for input costs and charges for services. 
Table 7. Dominica: Selected Industrial Production, 2000-2004

(Volume in metric tons, unless otherwise specified; value in thousands of Eastern Caribbean dollars)

\begin{tabular}{|c|c|c|c|c|c|}
\hline & 2000 & 2001 & 2002 & 2003 & $\begin{array}{l}\text { Prel. } \\
2004 \\
\end{array}$ \\
\hline \multicolumn{6}{|c|}{ Laundry soap 1/ } \\
\hline Volume & 6,460 & 6,444 & 4,514 & 5,206 & 4,615 \\
\hline Value & 8,914 & 8,759 & 6,526 & 8,137 & 8,359 \\
\hline \multicolumn{6}{|c|}{ Toilet soap 1/ } \\
\hline Volume & 5,176 & 4,028 & 4,512 & 3,677 & 4,623 \\
\hline Value & 14,799 & 10,902 & 12,041 & 10,043 & 16,229 \\
\hline \multicolumn{6}{|c|}{ Dental cream } \\
\hline Volume & 1,698 & 1,562 & 1,305 & 1,468 & 1,392 \\
\hline Value & 11,428 & 10,064 & 7,788 & 8,775 & 8,774 \\
\hline \multicolumn{6}{|c|}{ Fine fabric detergent } \\
\hline Volume & 823 & 684 & 676 & 559 & 513 \\
\hline Value & 2,003 & 1,646 & 1,554 & 1,264 & 1,225 \\
\hline \multicolumn{6}{|l|}{ Hand dish } \\
\hline Volume & 998 & 707 & 742 & 759 & 737 \\
\hline Value & 1,711 & 1,120 & 1,196 & 1,253 & 1,506 \\
\hline \multicolumn{6}{|c|}{ Liquid cleaner/disinfectant } \\
\hline Volume & 1,954 & 1,433 & 1,662 & 1,669 & 1,751 \\
\hline Value & 3,491 & 2,237 & 2,546 & 2,552 & 3,120 \\
\hline \multicolumn{6}{|c|}{ Floor polish } \\
\hline Volume & 194 & 116 & 110 & 91 & 32 \\
\hline Value & 504 & 299 & 303 & 260 & 88 \\
\hline \multicolumn{6}{|l|}{ Others } \\
\hline Volume & 36 & 47 & 63 & 66 & 55 \\
\hline Value & 84 & 130 & 171 & 188 & 168 \\
\hline
\end{tabular}

Sources: Central Statistical Office, Ministry of Finance; and Dominica Coconut Products, Ltd.

1/ Laundry and toilet soap represent about 90 percent of total soap production. 
Table 8. Dominica: Consumer Price Indices, 2000-2004

\begin{tabular}{|c|c|c|c|c|c|}
\hline & 2000 & 2001 & 2002 & 2003 & 2004 \\
\hline \multicolumn{6}{|c|}{ (Index: January $2001=100)$} \\
\hline CPI (period average) & 98.9 & 100.4 & 100.6 & 102.1 & 104.60 \\
\hline Food & $\ldots$ & 100.9 & 102.4 & 102.9 & 105.7 \\
\hline Alcoholic beverages and tobacco & $\ldots$ & 100.4 & 101.5 & 98.6 & 97.7 \\
\hline Clothing and footwear & $\ldots$ & 98.9 & 98.0 & 96.9 & 97.1 \\
\hline Housing and utilities & $\ldots$ & 100.3 & 100.1 & 103.7 & 108.6 \\
\hline Fuel and light & $\ldots$ & 97.0 & 100.6 & 112.1 & 120.5 \\
\hline Medical and health expenses & $\ldots$ & 100.1 & 100.3 & 99.3 & 99.6 \\
\hline Household equipment and furniture & $\ldots$ & 100.0 & 100.1 & 99.8 & 98.4 \\
\hline Transportation & $\ldots$ & 101.8 & 98.1 & 99.2 & 101.2 \\
\hline Educational expenses & $\ldots$ & 100.1 & 104.1 & 109.3 & 113.1 \\
\hline Personal care services & $\ldots$ & 100.0 & 101.5 & 104.5 & 105.7 \\
\hline Miscellaneous & $\ldots$ & 100.7 & 102.1 & 104.7 & 107.5 \\
\hline CPI (end of period) & 99.5 & 100.6 & 101.0 & 104.0 & 104.76 \\
\hline Food & $\ldots$ & 102.2 & 102.5 & 104.8 & 106.2 \\
\hline Alcoholic beverages and tobacco & $\ldots$ & 101.6 & 101.4 & 97.1 & 98.4 \\
\hline Clothing and footwear & $\ldots$ & 98.3 & 97.9 & 96.5 & 96.8 \\
\hline Housing and utilities & $\ldots$ & 100.0 & 100.0 & 107.1 & 110.6 \\
\hline Fuel and light & $\ldots$ & 89.9 & 105.7 & 112.9 & 112.8 \\
\hline Medical and health expenses & $\ldots$ & 100.1 & 100.5 & 99.5 & 99.8 \\
\hline Household equipment and furniture & $\ldots$ & 100.2 & 100.0 & 99.6 & 98.1 \\
\hline Transportation & $\ldots$ & 103.1 & 98.1 & 102.5 & 102.4 \\
\hline Educational expenses & $\begin{array}{l}\cdots \\
\cdots\end{array}$ & 100.1 & 105.4 & 112.1 & 113.8 \\
\hline Personal care services & $\ldots$ & 100.0 & 103.6 & 105.6 & 105.7 \\
\hline Miscellaneous & $\ldots$ & 101.4 & 102.8 & 107.5 & 107.8 \\
\hline \multicolumn{6}{|c|}{ (Percentage change) } \\
\hline Average & $\ldots$ & 1.6 & 0.1 & 1.6 & 2.4 \\
\hline Food & $\ldots$ & $\ldots$ & 1.5 & 0.4 & 2.8 \\
\hline Alcoholic beverages and tobacco & $\ldots$ & $\ldots$ & 1.1 & -2.9 & -0.9 \\
\hline Clothing and footwear & $\begin{array}{l}\cdots \\
\cdots\end{array}$ & $\cdots$ & -1.0 & -1.1 & 0.2 \\
\hline Housing and utilities & $\ldots$ & $\ldots$ & -0.3 & 3.6 & 4.7 \\
\hline Fuel and light & $\ldots$ & $\ldots$ & 3.7 & 11.4 & 7.5 \\
\hline Medical and health expenses & $\ldots$ & $\ldots$ & 0.2 & -0.9 & 0.3 \\
\hline Household equipment and furniture & $\ldots$ & $\ldots$ & 0.1 & -0.3 & -1.4 \\
\hline Transportation & $\ldots$ & $\ldots$ & -3.7 & 1.2 & 2.0 \\
\hline Educational expenses & $\ldots$ & $\ldots$ & 4.0 & 5.0 & 3.5 \\
\hline Personal care services & $\ldots$ & $\ldots$ & 1.5 & 3.0 & 1.1 \\
\hline Miscellaneous & $\ldots$ & $\ldots$ & 1.5 & 2.5 & 2.6 \\
\hline End of period & $\ldots$ & 1.1 & 0.4 & 2.9 & 0.8 \\
\hline Food & $\ldots$ & $\ldots$ & 0.4 & 2.2 & 1.3 \\
\hline Alcoholic beverages and tobacco & $\ldots$ & $\ldots$ & -0.2 & -4.2 & 1.3 \\
\hline Clothing and footwear & $\ldots$ & $\ldots$ & -0.5 & -1.4 & 0.3 \\
\hline Housing and utilities & $\ldots$ & $\ldots$ & 0.0 & 7.1 & 3.3 \\
\hline Fuel and light & $\ldots$ & $\ldots$ & 17.6 & 6.8 & -0.1 \\
\hline Medical and health expenses & $\ldots$ & $\ldots$ & 0.4 & -1.0 & 0.3 \\
\hline Household equipment and furniture & $\ldots$ & $\ldots$ & -0.1 & -0.5 & -1.5 \\
\hline Transportation & $\ldots$ & $\ldots$ & -4.8 & 4.5 & -0.2 \\
\hline Educational expenses & $\ldots$ & $\ldots$ & 5.3 & 6.3 & 1.5 \\
\hline Personal care services & $\ldots$ & $\ldots$ & 3.6 & 1.9 & 0.1 \\
\hline Miscellaneous & $\ldots$ & $\ldots$ & 1.3 & 4.6 & 0.3 \\
\hline
\end{tabular}

Source: Central Statistical Office, Ministry of Finance. 
Table 9. Dominica: Petroleum Imports and Retail Prices, 2000-2004 1/

\begin{tabular}{|c|c|c|c|c|c|}
\hline & 2000 & 2001 & 2002 & 2003 & 2004 \\
\hline \multicolumn{6}{|l|}{ Gasoline } \\
\hline Volume of imports (imperial gallons) & $4,037,861$ & $4,027,900$ & $3,728,387$ & $3,883,366$ & $3,894,253$ \\
\hline Average retail price (EC\$ per imperial gallon) & 6.90 & 6.90 & 7.15 & 7.98 & 8.85 \\
\hline \multicolumn{6}{|l|}{ Diesel } \\
\hline Volume of imports (imperial gallons) & $4,409,451$ & $4,520,999$ & $3,586,505$ & $4,799,556$ & $4,924,988$ \\
\hline Average retail price (EC\$ per imperial gallon) & 5.58 & 5.58 & 5.78 & 6.79 & 7.74 \\
\hline \multicolumn{6}{|l|}{ Kerosene } \\
\hline Volume of imports (imperial gallons) & 300,770 & 200,461 & 382,107 & 201,300 & 211,364 \\
\hline Average retail price (EC\$ per imperial gallon) & 4.35 & 5.22 & 5.22 & 5.22 & 6.43 \\
\hline \multicolumn{6}{|l|}{ Liquefied petroleum gas (LPG) } \\
\hline Volume of imports (kgs) & $2,569,210$ & $2,006,949$ & $2,008,335$ & $2,186,738$ & $2,284,618$ \\
\hline Average retail price (EC\$ per $20 \mathrm{lb}$. cylinder) & 29.30 & 29.30 & 30.33 & 31.36 & 31.36 \\
\hline Total value of petroleum imports (EC\$ millions) & 33.87 & 30.96 & 26.13 & 34.28 & 38.95 \\
\hline
\end{tabular}

Source: Central Statistical Office, Ministry of Finance.

$1 /$ The average retail price is calculated as the average of the end-month retail prices. 
Table 10. Dominica: Summary Accounts of the Consolidated Public Sector, 1999/00-2003/04 1/

\begin{tabular}{|c|c|c|c|c|c|}
\hline & $\begin{array}{r}\text { Est. } \\
1999 / 2000 \\
\end{array}$ & $\begin{array}{r}\text { Est. } \\
2000 / 01 \\
\end{array}$ & $\begin{array}{r}\text { Est. } \\
2001 / 02 \\
\end{array}$ & $\begin{array}{r}\text { Est. } \\
2002 / 03\end{array}$ & $\begin{array}{r}\text { Est. } \\
2003 / 04 \\
\end{array}$ \\
\hline \multicolumn{6}{|c|}{ (In millions of Eastern Caribbean dollars) } \\
\hline \multicolumn{6}{|c|}{ I. Consolidated Public Sector } \\
\hline Total revenue and grants & 329.3 & 377.8 & 302.1 & 307.5 & 376.3 \\
\hline Current revenue & 307.1 & 289.3 & 288.2 & 275.2 & 311.2 \\
\hline Capital revenue & 2.6 & 3.0 & 0.9 & 1.3 & 1.0 \\
\hline Total grants & 19.7 & 85.5 & 13.0 & 30.9 & 64.1 \\
\hline Total expenditure 2/ & 384.6 & 451.8 & 352.9 & 328.5 & 370.8 \\
\hline Current & 305.2 & 305.0 & 298.5 & 290.6 & 287.5 \\
\hline Wages and salaries & 121.6 & 122.5 & 126.3 & 123.1 & 111.5 \\
\hline Interest & 30.8 & 38.9 & 40.0 & 39.7 & 36.9 \\
\hline Transfers & 52.1 & 58.2 & 56.7 & 59.7 & 61.8 \\
\hline Other & 100.7 & 85.4 & 75.5 & 68.1 & 77.3 \\
\hline Capital expenditure & 79.3 & 146.8 & 54.4 & 37.9 & 83.3 \\
\hline Current account balance & 1.8 & -15.7 & -10.3 & -15.4 & 23.7 \\
\hline Overall balance (before grants) & -75.0 & -159.5 & -63.8 & -51.9 & -58.6 \\
\hline Overall balance & -55.3 & -74.0 & -50.8 & -21.0 & 5.5 \\
\hline Statistical discrepancy $3 /$ & -7.8 & 18.7 & 8.2 & -11.5 & 7.7 \\
\hline Financing (net) & 63.0 & 55.3 & 42.6 & 32.5 & -13.2 \\
\hline \multicolumn{6}{|c|}{ II. Central Government } \\
\hline Total revenue and grants & 233.7 & 271.5 & 209.7 & 224.1 & 285.9 \\
\hline Current revenue & 211.4 & 200.5 & 197.0 & 191.9 & 221.8 \\
\hline Tax & 178.4 & 169.6 & 160.2 & 167.9 & 193.6 \\
\hline Nontax & 33.0 & 31.0 & 36.8 & 23.9 & 28.1 \\
\hline Capital revenue & 2.6 & 3.0 & 0.9 & 1.3 & 1.0 \\
\hline Grants & 19.7 & 68.0 & 11.8 & 30.9 & 63.2 \\
\hline Total expenditure 2/ & 316.1 & 350.3 & 269.3 & 261.3 & 287.6 \\
\hline Current expenditure & 218.9 & 230.1 & 229.7 & 226.6 & 215.5 \\
\hline Wages and salaries & 112.6 & 116.2 & 116.5 & 116.1 & 104.2 \\
\hline Goods and services & 42.5 & 37.5 & 38.7 & 33.7 & 38.1 \\
\hline Interest & 28.0 & 36.0 & 36.9 & 37.6 & 34.1 \\
\hline Transfers & 35.7 & 40.4 & 37.6 & 39.1 & 39.1 \\
\hline Capital expenditure and net lending & 97.3 & 120.2 & 39.6 & 34.7 & 72.1 \\
\hline Fixed investment and capital transfers & 76.5 & 110.9 & 41.9 & 35.0 & 75.0 \\
\hline Net lending & 20.8 & 9.3 & -2.4 & -0.3 & -2.8 \\
\hline Current account balance & -7.4 & -29.6 & -32.7 & -34.7 & 6.3 \\
\hline Overall balance (before grants) & -102.1 & -146.8 & -71.4 & -68.1 & -64.9 \\
\hline Overall balance & -82.4 & -78.8 & -59.6 & -37.2 & -1.7 \\
\hline Statistical discrepancy 3/ & -7.8 & 18.7 & 8.2 & -11.5 & 7.7 \\
\hline Financing (net) & 90.2 & 60.1 & 51.3 & 48.7 & -6.0 \\
\hline External & 88.0 & 42.7 & 25.6 & 44.9 & 47.4 \\
\hline Domestic & 2.2 & 17.4 & 25.7 & 3.8 & -53.4 \\
\hline
\end{tabular}


Table 10. Dominica: Summary Accounts of the Consolidated Public Sector, 1999/00-2003/04 1/

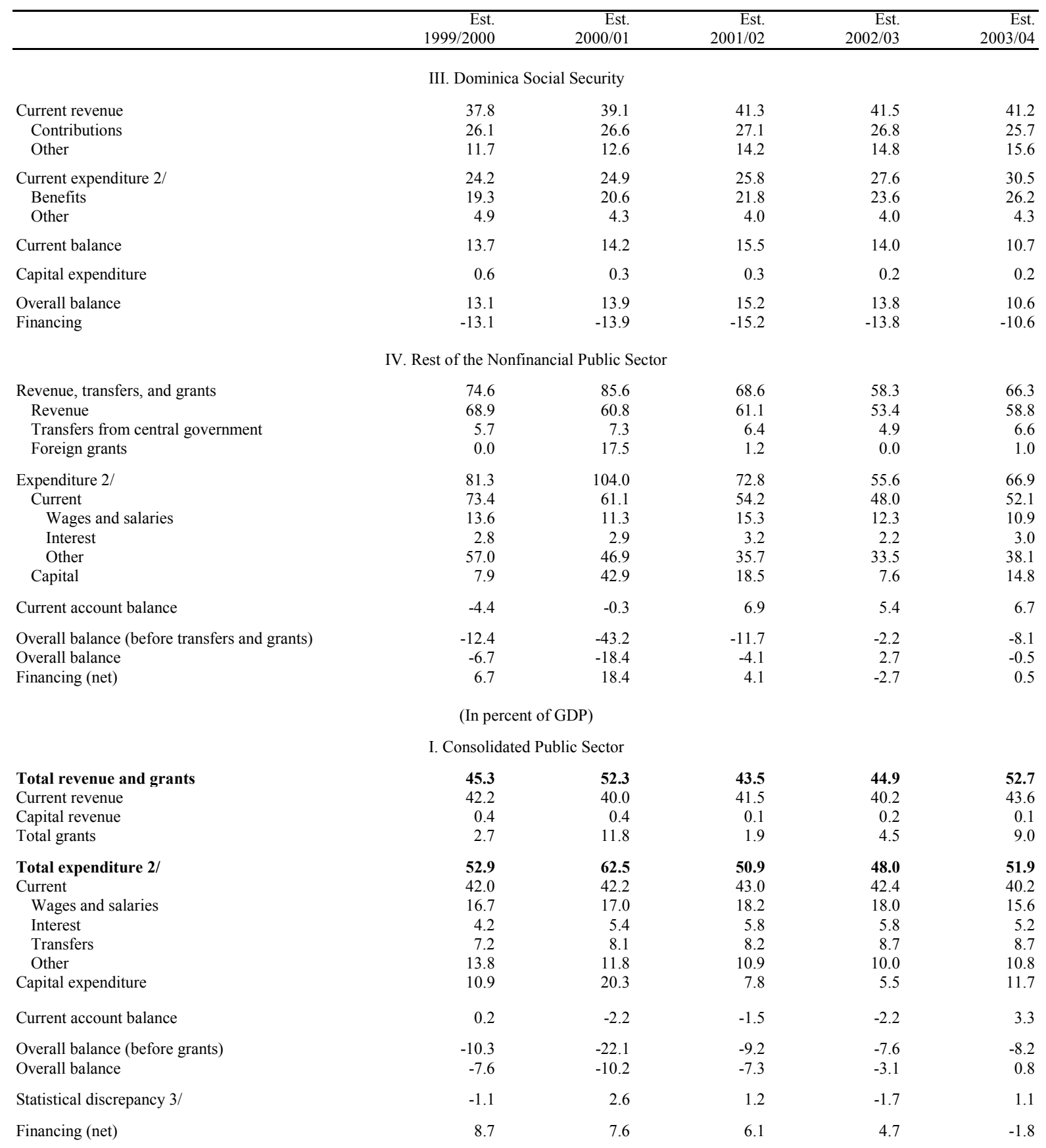


Table 10. Dominica: Summary Accounts of the Consolidated Public Sector, 1999/00-2003/04 1/

\begin{tabular}{|c|c|c|c|c|c|}
\hline & $\begin{array}{r}\text { Est. } \\
1999 / 2000 \\
\end{array}$ & $\begin{array}{r}\text { Est. } \\
2000 / 01 \\
\end{array}$ & $\begin{array}{r}\text { Est. } \\
2001 / 02 \\
\end{array}$ & $\begin{array}{r}\text { Est. } \\
2002 / 03 \\
\end{array}$ & $\begin{array}{r}\text { Est. } \\
2003 / 04 \\
\end{array}$ \\
\hline \multicolumn{6}{|c|}{ II. Central Government } \\
\hline Total revenue and grants & 32.1 & 37.6 & 30.2 & 32.7 & 40.0 \\
\hline Current revenue & 29.1 & 27.8 & 28.4 & 28.0 & 31.0 \\
\hline Tax & 24.5 & 23.5 & 23.1 & 24.5 & 27.1 \\
\hline Nontax & 4.5 & 4.3 & 5.3 & 3.5 & 3.9 \\
\hline Capital revenue & 0.4 & 0.4 & 0.1 & 0.2 & 0.1 \\
\hline Grants & 2.7 & 9.4 & 1.7 & 4.5 & 8.8 \\
\hline Total expenditure $2 /$ & 43.5 & 48.5 & 38.8 & 38.2 & 40.3 \\
\hline Current expenditure & 30.1 & 31.9 & 33.1 & 33.1 & 30.2 \\
\hline Wages and salaries & 15.5 & 16.1 & 16.8 & 17.0 & 14.6 \\
\hline Goods and services & 5.8 & 5.2 & 5.6 & 4.9 & 5.3 \\
\hline Interest & 3.9 & 5.0 & 5.3 & 5.5 & 4.8 \\
\hline Transfers & 4.9 & 5.6 & 5.4 & 5.7 & 5.5 \\
\hline Capital expenditure and net lending & 13.4 & 16.6 & 5.7 & 5.1 & 10.1 \\
\hline Fixed investment and capital transfers & 10.5 & 15.3 & 6.0 & 5.1 & 10.5 \\
\hline Net lending & 2.9 & 1.3 & -0.3 & 0.0 & -0.4 \\
\hline Current account balance & -1.0 & -4.1 & -4.7 & -5.1 & 0.9 \\
\hline Overall balance (before grants) & -14.0 & -20.3 & -10.3 & -9.9 & -9.1 \\
\hline Overall balance & -11.3 & -10.9 & -8.6 & -5.4 & -0.2 \\
\hline Statistical discrepancy $3 /$ & -1.1 & 2.6 & 1.2 & -1.7 & 1.1 \\
\hline Financing (net) & 12.4 & 8.3 & 7.4 & 7.1 & -0.8 \\
\hline External & 12.1 & 5.9 & 3.7 & 6.6 & 6.6 \\
\hline Domestic & 0.3 & 2.4 & 3.7 & 0.6 & -7.5 \\
\hline \multicolumn{6}{|c|}{ III. Dominica Social Security } \\
\hline Current revenue & 5.2 & 5.4 & 6.0 & 6.1 & 5.8 \\
\hline Contributions & 3.6 & 3.7 & 3.9 & 3.9 & 3.6 \\
\hline Other & 1.6 & 1.7 & 2.0 & 2.2 & 2.2 \\
\hline Current expenditure 2/ & 3.3 & 3.5 & 3.7 & 4.0 & 4.3 \\
\hline Benefits & 2.6 & 2.9 & 3.1 & 3.4 & 3.7 \\
\hline Other & 0.7 & 0.6 & 0.6 & 0.6 & 0.6 \\
\hline Current balance & 1.9 & 2.0 & 2.2 & 2.0 & 1.5 \\
\hline Capital expenditure & 0.1 & 0.0 & 0.0 & 0.0 & 0.0 \\
\hline Overall balance & 1.8 & 1.9 & 2.2 & 2.0 & 1.5 \\
\hline Financing & -1.8 & -1.9 & -2.2 & -2.0 & -1.5 \\
\hline \multicolumn{6}{|c|}{ IV. Rest of the Nonfinancial Public Sector } \\
\hline Revenue, transfers, and grants & 10.3 & 11.8 & 9.9 & 8.5 & 9.3 \\
\hline Revenue & 9.5 & 8.4 & 8.8 & 7.8 & 8.2 \\
\hline Transfers from central government & 0.8 & 1.0 & 0.9 & 0.7 & 0.9 \\
\hline Foreign grants & 0.0 & 2.4 & 0.2 & 0.0 & 0.1 \\
\hline Expenditure 2/ & 11.2 & 14.4 & 10.5 & 8.1 & 9.4 \\
\hline Current & 10.1 & 8.5 & 7.8 & 7.0 & 7.3 \\
\hline Wages and salaries & 1.9 & 1.6 & 2.2 & 1.8 & 1.5 \\
\hline Interest & 0.4 & 0.4 & 0.5 & 0.3 & 0.4 \\
\hline Other & 7.8 & 6.5 & 5.2 & 4.9 & 5.3 \\
\hline Capital & 1.1 & 5.9 & 2.7 & 1.1 & 2.1 \\
\hline Current account balance & -0.6 & 0.0 & 1.0 & 0.8 & 0.9 \\
\hline Overall balance (before transfers and grants) & -1.7 & -6.0 & -1.7 & -0.3 & -1.1 \\
\hline Overall balance & -0.9 & -2.6 & -0.6 & 0.4 & -0.1 \\
\hline Financing (net) & 0.9 & 2.6 & 0.6 & -0.4 & 0.1 \\
\hline
\end{tabular}

Sources: Dominican authorities; and Fund staff estimates.

1/ Fiscal years ending June 30.

$2 /$ On a cash basis.

3/ Difference between identified financing and overall balance. 
Table 11. Dominica: Summary Accounts of the Central Government, 2000/01-2004/05 1/

\begin{tabular}{|c|c|c|c|c|c|}
\hline & $2000 / 01$ & $2001 / 02$ & $2002 / 03$ & $2003 / 04$ & $2004 / 05$ \\
\hline \multicolumn{6}{|c|}{ (In millions of Eastern Caribbean dollars) } \\
\hline Total revenue and grants & 271.5 & 209.7 & 224.1 & 285.9 & 292.0 \\
\hline Current revenue & 200.5 & 197.1 & 191.9 & 221.8 & 245.7 \\
\hline Tax revenue & 169.6 & 160.2 & 167.9 & 193.6 & 213.3 \\
\hline Taxes on income & 54.6 & 40.1 & 45.0 & 43.7 & 40.8 \\
\hline Taxes on property & 2.6 & 2.2 & 2.5 & 2.6 & 6.6 \\
\hline Taxes on goods and services & 28.6 & 30.2 & 33.0 & 49.1 & 56.2 \\
\hline Taxes on international transactions & 83.8 & 87.6 & 87.5 & 98.3 & 109.7 \\
\hline Nontax revenue & 31.0 & 36.8 & 23.9 & 28.1 & 32.4 \\
\hline Capital revenue & 3.0 & 0.9 & 1.3 & 1.0 & 2.0 \\
\hline Foreign grants & 68.0 & 11.8 & 30.9 & 63.2 & 44.3 \\
\hline Total expenditure 2/ & 350.3 & 269.3 & 261.3 & 287.6 & 281.7 \\
\hline Current expenditure & 230.1 & 229.7 & 226.6 & 215.5 & 218.2 \\
\hline Wages and salaries & 116.2 & 116.5 & 116.1 & 104.2 & 102.0 \\
\hline Goods and services & 37.5 & 38.7 & 33.7 & 38.1 & 39.0 \\
\hline Interest & 36.0 & 36.9 & 37.6 & 34.1 & 29.2 \\
\hline Domestic & 20.2 & 19.3 & 17.8 & 17.0 & 8.1 \\
\hline External & 15.7 & 17.5 & 19.8 & 17.1 & 21.1 \\
\hline Retirement benefits & 13.4 & 15.9 & 16.5 & 18.9 & 21.5 \\
\hline Other transfers & 27.0 & 21.6 & 22.6 & 20.2 & 26.5 \\
\hline Capital expenditure and net lending & 120.2 & 39.6 & 34.7 & 72.1 & 63.5 \\
\hline Capital expenditure & 110.9 & 41.9 & 35.0 & 75.0 & 67.1 \\
\hline Net lending & 9.3 & -2.4 & -0.3 & -2.8 & -3.6 \\
\hline Current account balance & -29.6 & -32.6 & -34.7 & 6.3 & 27.5 \\
\hline Overall balance (before grants) & -146.8 & -71.3 & -68.1 & -64.9 & -34.0 \\
\hline Overall balance & -78.8 & -59.5 & -37.2 & -1.7 & 10.4 \\
\hline Statistical discrepancy $3 /$ & 18.7 & 8.2 & -11.5 & 7.7 & -7.0 \\
\hline Financing 2/ & 60.1 & 51.3 & 48.7 & -6.0 & -3.4 \\
\hline External & 42.7 & 25.6 & 44.9 & 47.4 & 17.2 \\
\hline Domestic & 17.4 & 25.7 & 3.8 & -53.4 & -20.6 \\
\hline Bank & 11.2 & 16.3 & -6.9 & -41.4 & -15.3 \\
\hline Nonbank & 6.2 & 9.5 & 10.7 & -15.9 & -4.3 \\
\hline Other & 0.0 & 0.0 & 0.0 & 3.9 & -0.9 \\
\hline
\end{tabular}


Table 11. Dominica: Summary Accounts of the Central Government, 2000/01-2004/05 1/

\begin{tabular}{|c|c|c|c|c|c|}
\hline & $2000 / 01$ & $2001 / 02$ & $2002 / 03$ & $2003 / 04$ & $2004 / 05$ \\
\hline \multicolumn{6}{|c|}{ (In percent of GDP) } \\
\hline Total revenue and grants & 37.6 & 30.2 & 32.7 & 40.0 & 39.1 \\
\hline Current revenue & 27.8 & 28.4 & 28.0 & 31.0 & 32.9 \\
\hline Tax revenue & 23.5 & 23.1 & 24.5 & 27.1 & 28.5 \\
\hline Taxes on income & 7.6 & 5.8 & 6.6 & 6.1 & 5.5 \\
\hline Taxes on property & 0.4 & 0.3 & 0.4 & 0.4 & 0.9 \\
\hline Taxes on goods and services & 4.0 & 4.4 & 4.8 & 6.9 & 7.5 \\
\hline Taxes on international transactions & 11.6 & 12.6 & 12.8 & 13.8 & 14.7 \\
\hline Nontax revenue & 4.3 & 5.3 & 3.5 & 3.9 & 4.3 \\
\hline Capital revenue & 0.4 & 0.1 & 0.2 & 0.1 & 0.3 \\
\hline Foreign grants & 9.4 & 1.7 & 4.5 & 8.8 & 5.9 \\
\hline Total expenditure 2/ & 48.5 & 38.8 & 38.2 & 40.3 & 37.7 \\
\hline Current expenditure & 31.9 & 33.1 & 33.1 & 30.2 & 29.2 \\
\hline Wages and salaries & 16.1 & 16.8 & 17.0 & 14.6 & 13.6 \\
\hline Goods and services & 5.2 & 5.6 & 4.9 & 5.3 & 5.2 \\
\hline Interest & 5.0 & 5.3 & 5.5 & 4.8 & 3.9 \\
\hline Domestic & 2.8 & 2.8 & 2.6 & 2.4 & 1.1 \\
\hline External & 2.2 & 2.5 & 2.9 & 2.4 & 2.8 \\
\hline Retirement benefits & 1.9 & 2.3 & 2.4 & 2.6 & 2.9 \\
\hline Other transfers & 3.7 & 3.1 & 3.3 & 2.8 & 3.5 \\
\hline Capital expenditure and net lending & 16.6 & 5.7 & 5.1 & 10.1 & 8.5 \\
\hline Capital expenditure & 15.3 & 6.0 & 5.1 & 10.5 & 9.0 \\
\hline Net lending & 1.3 & -0.3 & 0.0 & -0.4 & -0.5 \\
\hline Current account balance & -4.1 & -4.7 & -5.1 & 0.9 & 3.7 \\
\hline Overall balance (before grants) & -20.3 & -10.3 & -9.9 & -9.1 & -4.5 \\
\hline Overall balance & -10.9 & -8.6 & -5.4 & -0.2 & 1.4 \\
\hline Statistical discrepancy 3/ & 2.6 & 1.2 & -1.7 & 1.1 & -0.9 \\
\hline Financing 2/ & 8.3 & 7.4 & 7.1 & -0.8 & -0.4 \\
\hline External & 5.9 & 3.7 & 6.6 & 6.6 & 2.3 \\
\hline Domestic & 2.4 & 3.7 & 0.6 & -7.5 & -2.7 \\
\hline Bank & 1.5 & 2.3 & -1.0 & -5.8 & -2.0 \\
\hline Nonbank & 0.9 & 1.4 & 1.6 & -2.2 & -0.6 \\
\hline Other & 0.0 & 0.0 & 0.0 & 0.5 & -0.1 \\
\hline
\end{tabular}

(In millions of Eastern Caribbean dollars)

Memorandum item:

Nominal GDP at current market prices

722.4

693.7

684.8

714.4

747.6

Sources: Ministry of Finance; and Fund staff estimates.

1/ Fiscal years ending June 30 .

2/ On a cash basis.

3/ Difference between identified financing and overall balance. 
Table 12. Dominica: Central Government Revenue and Grants, 2000/01-2004/05 1/

\begin{tabular}{|c|c|c|c|c|c|}
\hline & $2000 / 01$ & $2001 / 02$ & $2002 / 03$ & $2003 / 04$ & $2004 / 05$ \\
\hline \multicolumn{6}{|c|}{ (In millions of Eastern Caribbean dollars) } \\
\hline Total revenue and grants & 271.5 & 209.7 & 224.1 & 285.9 & 292.0 \\
\hline Current revenue & 200.5 & 197.1 & 191.9 & 221.8 & 245.7 \\
\hline Tax revenue & 169.6 & 160.2 & 167.9 & 193.6 & 213.3 \\
\hline Taxes on income & 54.6 & 40.1 & 45.0 & 43.7 & 40.8 \\
\hline Personal income & 31.8 & 29.5 & 28.1 & 27.3 & 28.6 \\
\hline Corporate income & 24.3 & 12.2 & 7.9 & 9.8 & 13.0 \\
\hline Income tax refunds & -1.5 & -1.5 & -1.3 & -1.4 & -1.5 \\
\hline Stabilization levy & 0.0 & 0.0 & 10.2 & 8.0 & 0.7 \\
\hline Taxes on property & 2.6 & 2.2 & 2.5 & 2.6 & 6.6 \\
\hline Taxes on goods and services & 28.6 & 30.2 & 33.0 & 49.1 & 56.2 \\
\hline Consumption tax & 4.7 & 4.9 & 5.1 & 5.5 & 4.8 \\
\hline Sales tax & 14.5 & 16.8 & 19.2 & 30.5 & 36.5 \\
\hline Stamp duty on receipts & 0.2 & 0.2 & 0.2 & 0.2 & 0.2 \\
\hline Hotel accommodation tax & 0.6 & 0.6 & 0.5 & 0.7 & 0.9 \\
\hline Licences & 8.6 & 7.8 & 8.0 & 12.2 & 13.9 \\
\hline Other & 0.0 & 0.0 & 0.0 & 0.0 & 0.0 \\
\hline Taxes on international transactions & 83.8 & 87.6 & 87.5 & 98.3 & 109.7 \\
\hline Taxes on imports & 75.1 & 79.7 & 78.0 & 87.0 & 96.1 \\
\hline Import duties & 22.7 & 20.2 & 20.4 & 21.9 & 27.3 \\
\hline Stamp duties and documentation fee & 0.0 & 0.1 & 0.0 & 0.0 & 0.0 \\
\hline Consumption tax on imports & 45.4 & 53.1 & 51.3 & 54.4 & 56.6 \\
\hline Customs service charge & 6.9 & 6.3 & 6.2 & 10.7 & 12.2 \\
\hline Taxes on exports & 0.1 & 0.1 & 0.2 & 0.1 & 0.2 \\
\hline Other taxes on international trade & 8.7 & 7.9 & 9.2 & 11.2 & 13.4 \\
\hline Cruiseship passenger tax & 2.7 & 2.0 & 1.4 & 2.8 & 2.9 \\
\hline Environmental surcharge & 2.8 & 2.7 & 4.6 & 5.0 & 6.0 \\
\hline Embarkation tax & 2.8 & 2.8 & 2.9 & 3.0 & 4.1 \\
\hline Security tax & 0.3 & 0.3 & 0.3 & 0.3 & 0.5 \\
\hline Nontax revenue & 31.0 & 36.8 & 23.9 & 28.1 & 32.4 \\
\hline Rents and interest & 6.0 & 3.0 & 2.2 & 3.9 & 4.8 \\
\hline Fees, fines and sales & 8.7 & 7.4 & 7.9 & 11.1 & 16.6 \\
\hline ECCB profits & 1.1 & 1.5 & 0.0 & 1.5 & 0.0 \\
\hline Economic citizenship program & 2.9 & 1.6 & 1.1 & 0.2 & 0.6 \\
\hline Other nontax revenue & 12.3 & 23.2 & 12.8 & 11.4 & 10.5 \\
\hline Capital revenue & 3.0 & 0.9 & 1.3 & 1.0 & 2.0 \\
\hline Land sales & 0.3 & 0.1 & 0.6 & 0.5 & 1.5 \\
\hline Sale of other assets & 0.0 & 0.0 & 0.0 & 0.5 & 0.5 \\
\hline Other capital revenue & 2.7 & 0.8 & 0.7 & 0.0 & 0.0 \\
\hline Grants & 68.0 & 11.8 & 30.9 & 63.2 & 44.3 \\
\hline
\end{tabular}


Table 12. Dominica: Central Government Revenue and Grants, 2000/01-2004/05 1/

\begin{tabular}{|c|c|c|c|c|c|}
\hline & $2000 / 01$ & $2001 / 02$ & $2002 / 03$ & $2003 / 04$ & $2004 / 05$ \\
\hline \multicolumn{6}{|c|}{ (In percent of GDP) } \\
\hline Total revenue and grants & 37.6 & 30.2 & 32.7 & 40.0 & 39.1 \\
\hline Current revenue & 27.8 & 28.4 & 28.0 & 31.0 & 32.9 \\
\hline Tax revenue & 23.5 & 23.1 & 24.5 & 27.1 & 28.5 \\
\hline Taxes on income & 7.6 & 5.8 & 6.6 & 6.1 & 5.5 \\
\hline Personal income & 4.4 & 4.2 & 4.1 & 3.8 & 3.8 \\
\hline Corporate income & 3.4 & 1.8 & 1.2 & 1.4 & 1.7 \\
\hline Income tax refunds & -0.2 & -0.2 & -0.2 & -0.2 & -0.2 \\
\hline Development levy & 0.0 & 0.0 & 1.5 & 1.1 & 0.1 \\
\hline Taxes on property & 0.4 & 0.3 & 0.4 & 0.4 & 0.9 \\
\hline Taxes on goods and services & 4.0 & 4.4 & 4.8 & 6.9 & 7.5 \\
\hline Consumption tax & 0.6 & 0.7 & 0.7 & 0.8 & 0.6 \\
\hline Sales tax & 2.0 & 2.4 & 2.8 & 4.3 & 4.9 \\
\hline Stamp duty on receipts & 0.0 & 0.0 & 0.0 & 0.0 & 0.0 \\
\hline Hotel accommodation tax & 0.1 & 0.1 & 0.1 & 0.1 & 0.1 \\
\hline Licences & 1.2 & 1.1 & 1.2 & 1.7 & 1.9 \\
\hline Other & 0.0 & 0.0 & 0.0 & 0.0 & 0.0 \\
\hline Taxes on international transactions & 11.6 & 12.6 & 12.8 & 13.8 & 14.7 \\
\hline Taxes on imports & 10.4 & 11.5 & 11.4 & 12.2 & 12.9 \\
\hline Import duties & 3.1 & 2.9 & 3.0 & 3.1 & 3.7 \\
\hline Stamp duties and documentation fee & 0.0 & 0.0 & 0.0 & 0.0 & 0.0 \\
\hline Consumption tax on imports & 6.3 & 7.6 & 7.5 & 7.6 & 7.6 \\
\hline Customs service charge & 1.0 & 0.9 & 0.9 & 1.5 & 1.6 \\
\hline Taxes on exports & 0.0 & 0.0 & 0.0 & 0.0 & 0.0 \\
\hline Other taxes on international trade & 1.2 & 1.1 & 1.3 & 1.6 & 1.8 \\
\hline Cruiseship passenger tax & 0.4 & 0.3 & 0.2 & 0.4 & 0.4 \\
\hline Environmental surcharge & 0.4 & 0.4 & 0.7 & 0.7 & 0.8 \\
\hline Embarkation tax & 0.4 & 0.4 & 0.4 & 0.4 & 0.5 \\
\hline Security tax & 0.0 & 0.0 & 0.0 & 0.0 & 0.1 \\
\hline Nontax revenue & 4.3 & 5.3 & 3.5 & 3.9 & 4.3 \\
\hline Rents and interest & 0.8 & 0.4 & 0.3 & 0.5 & 0.6 \\
\hline Fees, fines and sales & 1.2 & 1.1 & 1.1 & 1.6 & 2.2 \\
\hline ECCB profits & 0.2 & 0.2 & 0.0 & 0.2 & 0.0 \\
\hline Economic citizenship program & 0.4 & 0.2 & 0.2 & 0.0 & 0.1 \\
\hline Other nontax revenue & 1.7 & 3.3 & 1.9 & 1.6 & 1.4 \\
\hline Capital revenue & 0.4 & 0.1 & 0.2 & 0.1 & 0.3 \\
\hline Land sales & 0.0 & 0.0 & 0.1 & 0.1 & 0.2 \\
\hline Sale of other assets & 0.0 & 0.0 & 0.0 & 0.1 & 0.1 \\
\hline Other capital revenue & 0.4 & 0.1 & 0.1 & 0.0 & 0.0 \\
\hline Grants & 9.4 & 1.7 & 4.5 & 8.8 & 5.9 \\
\hline
\end{tabular}

Sources: Ministry of Finance; and Fund staff estimates.

1/ Fiscal years ending June 30. 
Table 13. Dominica: Central Government Expenditure, 2000/01-2004/05 1/2/

\begin{tabular}{|c|c|c|c|c|c|}
\hline & $2000 / 01$ & $2001 / 02$ & $2002 / 03$ & $2003 / 04$ & $2004 / 05$ \\
\hline \multicolumn{6}{|c|}{ (In millions of Eastern Caribbean dollars) } \\
\hline Total expenditure & 350.3 & 269.3 & 261.3 & 287.6 & 281.7 \\
\hline Current expenditure & 230.1 & 229.7 & 226.6 & 215.5 & 218.2 \\
\hline Wages and salaries & 116.2 & 116.5 & 116.1 & 104.2 & 102.0 \\
\hline Salaries & 106.8 & 109.6 & 107.3 & 98.0 & 95.7 \\
\hline Wages & 9.5 & 7.0 & 8.9 & 6.2 & 6.3 \\
\hline Goods and services & 37.5 & 38.7 & 33.7 & 38.1 & 39.0 \\
\hline Interest & 36.0 & 36.9 & 37.6 & 34.1 & 29.2 \\
\hline Domestic & 20.2 & 19.3 & 17.8 & 17.0 & 8.1 \\
\hline External & 15.7 & 17.5 & 19.8 & 17.1 & 21.1 \\
\hline Current transfers & 40.4 & 37.6 & 39.1 & 39.1 & 48.0 \\
\hline Retirement benefits & 13.4 & 15.9 & 16.5 & 18.9 & 21.5 \\
\hline Regional and international & 9.6 & 8.4 & 4.9 & 1.5 & 7.6 \\
\hline Public assistance and poverty relief & 2.5 & 3.2 & 3.0 & 3.1 & 3.3 \\
\hline Other & 14.9 & 10.0 & 14.6 & 15.6 & 15.6 \\
\hline Capital expenditure and net lending & 120.2 & 39.6 & 34.7 & 72.1 & 63.5 \\
\hline Capital expenditure & 110.9 & 41.9 & 35.0 & 75.0 & 67.1 \\
\hline Net lending & 9.3 & -2.4 & -0.3 & -2.8 & -3.6 \\
\hline \multicolumn{6}{|c|}{ (In percent of GDP) } \\
\hline Total expenditure & 48.5 & 38.8 & 38.2 & 40.3 & 37.7 \\
\hline Current expenditure & 31.9 & 33.1 & 33.1 & 30.2 & 29.2 \\
\hline Wages and salaries & 16.1 & 16.8 & 17.0 & 14.6 & 13.6 \\
\hline Salaries & 14.8 & 15.8 & 15.7 & 13.7 & 12.8 \\
\hline Wages & 1.3 & 1.0 & 1.3 & 0.9 & 0.8 \\
\hline Goods and services & 5.2 & 5.6 & 4.9 & 5.3 & 5.2 \\
\hline Interest & 5.0 & 5.3 & 5.5 & 4.8 & 3.9 \\
\hline Domestic & 2.8 & 2.8 & 2.6 & 2.4 & 1.1 \\
\hline External & 2.2 & 2.5 & 2.9 & 2.4 & 2.8 \\
\hline Current transfers & 5.6 & 5.4 & 5.7 & 5.5 & 6.4 \\
\hline Retirement benefits & 1.9 & 2.3 & 2.4 & 2.6 & 2.9 \\
\hline Regional and international & 1.3 & 1.2 & 0.7 & 0.2 & 1.0 \\
\hline Public assistance and poverty relief & 0.3 & 0.5 & 0.4 & 0.4 & 0.4 \\
\hline Other & 2.1 & 1.4 & 2.1 & 2.2 & 2.1 \\
\hline Capital expenditure and net lending & 16.6 & 5.7 & 5.1 & 10.1 & 8.5 \\
\hline Capital expenditure & 15.3 & 6.0 & 5.1 & 10.5 & 9.0 \\
\hline Net lending & 1.3 & -0.3 & 0.0 & -0.4 & -0.5 \\
\hline
\end{tabular}

Sources: Ministry of Finance; and Fund staff estimates.

1/ Fiscal years ending June 30.

2/ On a cash basis. 
Table 14. Dominica: Public Sector Investment, 2000/01-2004/05 1/

\begin{tabular}{|c|c|c|c|c|c|}
\hline & $2000 / 01$ & $2001 / 02$ & $2002 / 03$ & $2003 / 04$ & $2004 / 05$ \\
\hline \multicolumn{6}{|c|}{ (In millions of Eastern Caribbean dollars) } \\
\hline Central government investment program & 110.9 & 41.9 & 35.0 & 75.0 & 67.1 \\
\hline Economic infrastructure & 28.6 & 22.7 & 18.2 & 23.5 & 32.5 \\
\hline Airport development & 0.0 & 0.0 & 0.0 & 0.8 & 4.6 \\
\hline Roads and bridges & 7.6 & 14.7 & 11.8 & 9.7 & 15.0 \\
\hline Sea defences & 21.0 & 3.5 & 3.0 & 12.7 & 3.7 \\
\hline Water and sewerage & 0.0 & 0.0 & 0.2 & 0.0 & 0.0 \\
\hline Other economic infrastructure & 0.0 & 4.5 & 3.2 & 0.3 & 9.2 \\
\hline Productive sectors & 4.6 & 0.9 & 1.8 & 34.6 & 17.3 \\
\hline Agriculture & 1.2 & 0.9 & 1.7 & 2.4 & 8.4 \\
\hline Forestry & 0.0 & 0.0 & 0.0 & 0.0 & 0.0 \\
\hline Micro-enterprise development & 1.3 & 0.0 & 0.0 & 0.1 & 0.2 \\
\hline Tourism development & 1.3 & 0.0 & 0.1 & 4.6 & 8.7 \\
\hline Other productive sectors & 0.8 & 0.0 & 0.0 & 27.5 & 0.0 \\
\hline Public administration & 32.8 & 1.2 & 0.6 & 6.8 & 3.6 \\
\hline Administrative infrastructure & 4.1 & 0.4 & 0.0 & 0.3 & 1.1 \\
\hline Environmental management & 0.0 & 0.0 & 0.2 & 0.3 & 0.5 \\
\hline Information systems development & 2.1 & 0.6 & 0.4 & 1.3 & 0.2 \\
\hline Offshore investment & 22.1 & 0.0 & 0.0 & 0.0 & 0.0 \\
\hline Public safety & 4.5 & 0.1 & 0.0 & 3.5 & 1.1 \\
\hline Public sector modernization & 0.0 & 0.1 & 0.0 & 1.4 & 0.7 \\
\hline Social infrastructure & 44.9 & 13.0 & 3.5 & 6.0 & 13.4 \\
\hline Community development & 5.1 & 1.4 & 0.0 & 1.4 & 2.5 \\
\hline Education & 14.3 & 9.4 & 1.3 & 3.6 & 4.6 \\
\hline Health & 2.3 & 0.3 & 0.4 & 1.0 & 2.2 \\
\hline Housing and settlement & 2.2 & 0.8 & 0.2 & 0.0 & 0.6 \\
\hline Sports and recreation & 21.0 & 1.1 & 1.5 & 0.0 & 3.5 \\
\hline Unclassified & 0.0 & 4.1 & 10.9 & 4.1 & 0.3 \\
\hline Financing of central government investment program & 110.9 & 41.9 & 35.0 & 75.0 & 67.1 \\
\hline Grants & 35.7 & 11.8 & 25.3 & 52.7 & 41.7 \\
\hline Loans & 69.3 & 13.0 & 3.0 & 20.2 & 18.6 \\
\hline Local counterpart & 5.9 & 17.1 & 6.7 & 2.1 & 6.8 \\
\hline Rest of public sector fixed investment $2 /$ & 43.2 & 18.8 & 7.8 & 15.0 & $\ldots$ \\
\hline \multicolumn{6}{|c|}{ (In percent of GDP) } \\
\hline Central government investment program & 15.4 & 6.0 & 5.1 & 10.5 & 9.4 \\
\hline Economic infrastructure & 4.0 & 3.3 & 2.7 & 3.3 & 4.5 \\
\hline Productive sectors & 0.6 & 0.1 & 0.3 & 4.8 & 2.4 \\
\hline Public administration & 4.5 & 0.2 & 0.1 & 1.0 & 0.5 \\
\hline Social infrastructure & 6.2 & 1.9 & 0.5 & 0.8 & 1.9 \\
\hline Unclassified & 0.0 & 0.6 & 1.6 & 0.6 & 0.0 \\
\hline Financing of central government investment program & 15.4 & 6.0 & 5.1 & 10.5 & 9.4 \\
\hline Grants & 4.9 & 1.7 & 3.7 & 7.4 & 5.8 \\
\hline Loans & 9.6 & 1.9 & 0.4 & 2.8 & 2.6 \\
\hline Local counterpart & 0.8 & 2.5 & 1.0 & 0.3 & 1.0 \\
\hline Rest of public sector fixed investment $2 /$ & 6.0 & 2.7 & 1.1 & 2.1 & $\ldots$ \\
\hline
\end{tabular}

Sources: Dominican authorities; and Fund staff estimates.

1/ Fiscal years ending June 30 .

2/ Fixed investment of Dominica Social Security and of the nonfinancial public enterprises. 
Table 15. Dominica: Central Government Domestic Debt, 2001-2005

(In millions of Eastern Caribbean dollars)

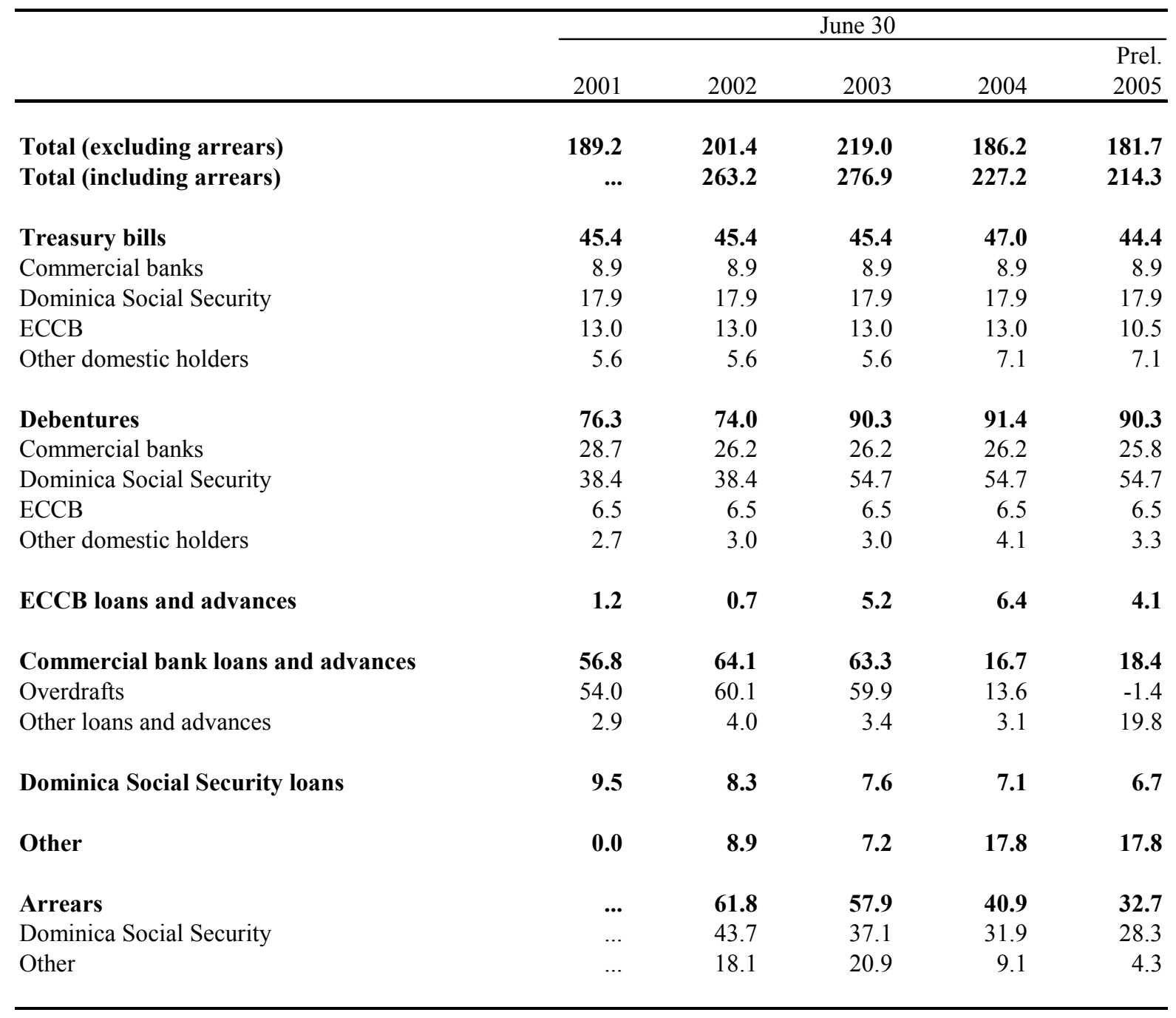

Sources: Dominican authorities; and Fund staff estimates. 
Table 16. Dominica: Consolidated Accounts of the Public Enterprises, 1999/00-2003/04 1/

\begin{tabular}{|c|c|c|c|c|c|}
\hline & $1999 / 2000$ & $2000 / 01$ & $2001 / 02$ & $\begin{array}{r}\text { Est. } \\
2002 / 03 \\
\end{array}$ & $\begin{array}{r}\text { Est. } \\
2003 / 04 \\
\end{array}$ \\
\hline \multicolumn{6}{|c|}{ (In millions of Eastern Caribbean dollars) } \\
\hline \multicolumn{6}{|c|}{ I. Consolidated Accounts } \\
\hline Current revenue & 68.9 & 60.8 & 61.1 & 53.4 & 58.8 \\
\hline Current expenditure $2 /$ & 73.4 & 61.1 & 54.2 & 48.0 & 52.1 \\
\hline Wages and salaries & 13.6 & 11.3 & 15.3 & 12.3 & 10.9 \\
\hline Interest & 2.8 & 2.9 & 3.2 & 2.2 & 3.0 \\
\hline Other & 57.0 & 46.9 & 35.7 & 33.5 & 38.1 \\
\hline Current balance & -4.4 & -0.3 & 6.9 & 5.4 & 6.7 \\
\hline Capital expenditure & 7.9 & 42.9 & 18.5 & 7.6 & 14.8 \\
\hline Overall balance (before transfers and grants) & -12.4 & -43.2 & -11.7 & -2.2 & -8.1 \\
\hline Transfers and grants & 5.7 & 24.8 & 7.5 & 4.9 & 7.6 \\
\hline Central government & 5.7 & 7.3 & 6.4 & 4.9 & 6.6 \\
\hline Foreign & 0.0 & 17.5 & 1.2 & 0.0 & 1.0 \\
\hline Overall balance & -6.7 & -18.4 & -4.1 & 2.7 & -0.5 \\
\hline Financing & 6.7 & 18.4 & 4.1 & -2.7 & 0.5 \\
\hline \multicolumn{6}{|c|}{ II. Dominica Water and Sewerage Authority } \\
\hline Current revenue & 12.0 & 11.9 & 12.5 & 13.0 & 12.5 \\
\hline Water sales & 11.3 & 11.3 & 11.4 & 11.8 & 11.7 \\
\hline Other & 0.7 & 0.7 & 1.1 & 1.2 & 0.7 \\
\hline Current expenditure $2 /$ & 7.3 & 6.9 & 6.7 & 7.4 & 8.6 \\
\hline Wages and salaries & 1.5 & 1.4 & 1.2 & 1.3 & 1.3 \\
\hline Interest & 0.5 & 0.5 & 0.6 & 0.7 & 1.7 \\
\hline Other & 5.4 & 5.0 & 4.9 & 5.5 & 5.6 \\
\hline Current balance & 4.6 & 5.1 & 5.8 & 5.5 & 3.9 \\
\hline Capital expenditure & 6.1 & 40.0 & 16.7 & 6.9 & 13.5 \\
\hline Overall balance (before transfers and grants) & -1.5 & -35.0 & -10.9 & -1.3 & -9.6 \\
\hline Transfers and grants & 0.1 & 17.6 & 1.2 & 0.2 & 4.9 \\
\hline Central government & 0.1 & 0.1 & 0.0 & 0.2 & 3.9 \\
\hline Foreign grants & 0.0 & 17.5 & 1.2 & 0.0 & 1.0 \\
\hline Overall balance & -1.4 & -17.3 & -9.7 & -1.1 & -4.7 \\
\hline Financing & 1.4 & 17.3 & 9.7 & 1.1 & 4.7 \\
\hline
\end{tabular}


Table 16. Dominica: Consolidated Accounts of the Public Enterprises, 1999/00-2003/04 1/

\begin{tabular}{|c|c|c|c|c|c|}
\hline & $1999 / 2000$ & $2000 / 01$ & $2001 / 02$ & $\begin{array}{r}\text { Est. } \\
2002 / 03 \\
\end{array}$ & $\begin{array}{r}\text { Est. } \\
2003 / 04 \\
\end{array}$ \\
\hline \multicolumn{6}{|c|}{ III. Dominica Export and Import Agency } \\
\hline Current revenue & 6.3 & 6.7 & 6.3 & 5.8 & 6.0 \\
\hline Current expenditure 2/ & 5.9 & 5.4 & 5.2 & 5.1 & 5.3 \\
\hline Wages and salaries & 0.5 & 0.5 & 0.6 & 0.5 & 0.5 \\
\hline Interest & 0.0 & 0.0 & 0.0 & 0.0 & 0.0 \\
\hline Other & 5.4 & 4.9 & 4.6 & 4.5 & 4.7 \\
\hline Current balance & 0.5 & 1.3 & 1.1 & 0.7 & 0.7 \\
\hline Capital expenditure & 0.2 & 0.3 & 0.1 & 0.2 & 0.1 \\
\hline Overall balance & 0.2 & 1.0 & 1.0 & 0.5 & 0.6 \\
\hline Financing & -0.2 & -1.0 & -1.0 & -0.5 & -0.6 \\
\hline \multicolumn{6}{|c|}{ IV. Dominica Port Authority } \\
\hline Current revenue & 12.3 & 11.8 & 14.8 & 13.6 & 14.0 \\
\hline Current expenditure $2 /$ & 8.7 & 9.1 & 13.4 & 12.5 & 10.4 \\
\hline Wages and salaries & 4.5 & 4.7 & 9.0 & 7.6 & 6.1 \\
\hline Interest & 1.4 & 1.2 & 1.3 & 1.3 & 1.1 \\
\hline Other & 2.8 & 3.2 & 3.1 & 3.6 & 3.1 \\
\hline Current balance & 3.6 & 2.7 & 1.4 & 1.2 & 3.6 \\
\hline Capital expenditure & 0.7 & 2.3 & 1.7 & 0.4 & 1.0 \\
\hline Overall balance & 3.0 & 0.3 & -0.3 & 0.8 & 2.6 \\
\hline Financing & -3.0 & -0.3 & 0.3 & -0.8 & -2.6 \\
\hline \multicolumn{6}{|c|}{ V. Dominica Broadcasting Corporation } \\
\hline Current revenue & 1.8 & 1.7 & 1.5 & 1.3 & 1.4 \\
\hline Current expenditure $2 /$ & 1.5 & 1.9 & 1.5 & 1.4 & 1.3 \\
\hline Wages and salaries & 0.9 & 1.0 & 0.9 & 0.7 & 0.7 \\
\hline Interest & 0.0 & 0.0 & 0.1 & 0.1 & 0.0 \\
\hline Other & 0.6 & 0.9 & 0.6 & 0.6 & 0.6 \\
\hline Current balance & 0.2 & -0.1 & 0.0 & -0.1 & 0.1 \\
\hline Capital expenditure & 0.2 & 0.2 & 0.0 & 0.1 & 0.1 \\
\hline Overall balance & 0.0 & -0.3 & 0.0 & -0.2 & -0.1 \\
\hline Financing & 0.0 & 0.3 & 0.0 & 0.2 & 0.1 \\
\hline
\end{tabular}


Table 16. Dominica: Consolidated Accounts of the Public Enterprises, 1999/00-2003/04 1/

\begin{tabular}{|c|c|c|c|c|c|}
\hline & $1999 / 2000$ & $2000 / 01$ & $2001 / 02$ & $\begin{array}{r}\text { Est. } \\
2002 / 03 \\
\end{array}$ & $\begin{array}{r}\text { Est. } \\
2003 / 04 \\
\end{array}$ \\
\hline \multicolumn{6}{|c|}{ VI. National Development Corporation } \\
\hline Current revenue & 0.7 & 0.2 & 0.7 & 0.7 & 0.8 \\
\hline Current expenditure $2 /$ & 4.3 & 3.8 & 2.9 & 2.8 & 2.6 \\
\hline Wages and salaries & 1.7 & 1.6 & 1.5 & 1.3 & 1.2 \\
\hline Interest & 0.0 & 0.0 & 0.0 & 0.0 & 0.0 \\
\hline Other & 2.7 & 2.2 & 1.4 & 1.4 & 1.4 \\
\hline Current balance & -3.7 & -3.6 & -2.2 & -2.0 & -1.9 \\
\hline Capital expenditure & 0.1 & 0.0 & 0.0 & 0.0 & 0.0 \\
\hline Transfers from central government & 4.1 & 3.7 & 3.0 & 4.7 & 2.7 \\
\hline Overall balance & 0.3 & 0.1 & 0.8 & 2.7 & 0.8 \\
\hline Financing & -0.3 & -0.1 & -0.8 & -2.7 & -0.8 \\
\hline \multicolumn{6}{|c|}{ VII. Dominica Banana Producers Limited 3/ } \\
\hline Current revenue & $\ldots$ & $\ldots$ & $\ldots$ & 18.9 & 24.2 \\
\hline Current expenditure $2 /$ & $\ldots$ & $\ldots$ & $\ldots$ & 18.8 & 23.9 \\
\hline Wages and salaries & $\ldots$ & $\ldots$ & $\ldots$ & 0.9 & 1.0 \\
\hline Interest & $\ldots$ & $\ldots$ & $\ldots$ & 0.2 & 0.2 \\
\hline Other & $\ldots$ & $\cdots$ & $\ldots$ & 17.8 & 22.7 \\
\hline Current balance & $\ldots$ & $\ldots$ & $\ldots$ & 0.1 & 0.3 \\
\hline Capital expenditure & $\ldots$ & $\ldots$ & $\ldots$ & 0.0 & 0.0 \\
\hline Overall balance & $\ldots$ & $\ldots$ & $\ldots$ & 0.1 & 0.3 \\
\hline Financing & $\ldots$ & $\ldots$ & $\ldots$ & -0.1 & -0.3 \\
\hline \multicolumn{6}{|c|}{ VIII. Dominica Banana Marketing Corporation 3/ } \\
\hline Revenue & 35.9 & 28.4 & 25.2 & $\ldots$ & $\ldots$ \\
\hline Banana sales & 34.4 & 25.0 & 20.2 & $\cdots$ & $\ldots$ \\
\hline Other income & 1.5 & 3.4 & 5.0 & $\ldots$ & $\ldots$ \\
\hline Current expenditure 2/ & 45.6 & 33.9 & 24.5 & $\ldots$ & $\ldots$ \\
\hline Wages and salaries & 4.5 & 2.1 & 2.1 & $\ldots$ & $\ldots$ \\
\hline Interest & 0.9 & 1.1 & 1.2 & $\ldots$ & $\ldots$ \\
\hline Other & 40.2 & 30.7 & 21.2 & $\cdots$ & $\ldots$ \\
\hline Current balance & -9.7 & -5.5 & 0.7 & $\ldots$ & ... \\
\hline Capital expenditure & 0.6 & 0.1 & 0.0 & $\ldots$ & $\ldots$ \\
\hline Transfer from central government & 1.5 & 3.4 & 3.3 & $\ldots$ & $\ldots$ \\
\hline Overall balance & -8.8 & -2.2 & 4.0 & $\ldots$ & $\ldots$ \\
\hline Financing & 8.8 & 2.2 & -4.0 & $\ldots$ & $\ldots$ \\
\hline
\end{tabular}

Sources: Ministry of Finance; and Fund staff estimates.

1/ Fiscal years ending June 30.

2/ On a cash basis.

3/ Source data are calendar year. To facilitate comparison, they have been converted to fiscal years by averaging. 
Table 17. Dominica: Monetary Survey, 2000-2004 1/

\begin{tabular}{|c|c|c|c|c|c|}
\hline & 2000 & 2001 & 2002 & 2003 & $\begin{array}{l}\text { Prel. } \\
2004 \\
\end{array}$ \\
\hline \multicolumn{6}{|c|}{ (In millions of Eastern Caribbean dollars; end of period) } \\
\hline Net foreign assets & 65.2 & 96.1 & 193.8 & 289.0 & 333.7 \\
\hline Monetary authorities & 78.2 & 82.1 & 114.9 & 118.7 & 90.8 \\
\hline Commercial banks & -12.9 & 14.0 & 79.0 & 170.4 & 242.9 \\
\hline Net domestic assets & 405.8 & 409.8 & 355.4 & 265.5 & 253.6 \\
\hline Domestic credit & 493.2 & 503.4 & 460.6 & 389.9 & 391.9 \\
\hline Net credit to the NFPS & 74.9 & 101.4 & 74.0 & 51.1 & 23.0 \\
\hline Central government & 70.3 & 92.3 & 64.2 & 55.9 & 45.6 \\
\hline Social Security & -14.3 & -10.8 & -9.0 & -21.2 & -34.8 \\
\hline Public enterprises & 18.9 & 19.9 & 18.7 & 16.5 & 12.1 \\
\hline Net credit to nonbank financial institutions & -35.8 & -37.7 & -46.6 & -81.8 & -75.9 \\
\hline Private sector credit & 454.1 & 439.6 & 433.2 & 420.6 & 444.7 \\
\hline Other items (net) & -87.4 & -93.5 & -105.2 & -124.5 & -138.2 \\
\hline Broad money & 471.0 & 506.0 & 549.2 & 554.5 & $\mathbf{5 8 7 . 4}$ \\
\hline Money & 90.3 & 91.6 & 106.6 & 107.9 & 111.2 \\
\hline Currency in circulation & 35.4 & 34.6 & 35.5 & 34.2 & 37.6 \\
\hline Demand deposits & 54.9 & 57.0 & 71.1 & 73.7 & 73.6 \\
\hline Quasi-money & 380.7 & 414.4 & 442.6 & 446.6 & 476.2 \\
\hline Savings deposits & 249.0 & 259.0 & 262.2 & 279.0 & 328.5 \\
\hline Time deposits & 121.7 & 139.5 & 158.2 & 157.4 & 134.3 \\
\hline Foreign currency deposits & 10.0 & 15.9 & 22.3 & 10.2 & 13.4 \\
\hline \multicolumn{6}{|c|}{ (Annual percentage change) } \\
\hline Broad money & 0.6 & 7.4 & 8.5 & 1.0 & 5.9 \\
\hline Money & -15.4 & 1.4 & 16.5 & 1.2 & 3.1 \\
\hline Quasi-money & 5.3 & 8.9 & 6.8 & 0.9 & 6.6 \\
\hline Credit to the private sector & 8.2 & -3.2 & -1.4 & -2.9 & 5.7 \\
\hline \multicolumn{6}{|c|}{ (Percentage change in terms of broad money 12 months earlier) } \\
\hline Net foreign assets & -12.8 & 6.6 & 19.3 & 17.3 & 8.1 \\
\hline Net domestic assets & 13.4 & 0.9 & -10.8 & -16.4 & -2.1 \\
\hline Net credit to the nonfinancial public sector & 5.7 & 5.6 & -5.4 & -4.2 & -5.1 \\
\hline Net credit to nonbank financial institutions & 1.7 & -0.4 & -1.8 & -6.4 & 1.1 \\
\hline Credit to the private sector & 7.3 & -3.1 & -1.3 & -2.3 & 4.3 \\
\hline Other items & -1.4 & -1.3 & -2.3 & -3.5 & -2.5 \\
\hline \multicolumn{6}{|l|}{ Memorandum item: } \\
\hline Income velocity of broad money & 1.54 & 1.40 & 1.24 & 1.26 & 1.25 \\
\hline
\end{tabular}

Sources: Eastern Caribbean Central Bank (ECCB); and Fund staff estimates.

1/ From 2002, transactions with the IMF are included as transactions with the monetary authorities. 
Table 18. Dominica: Balance Sheet of the Monetary Authorities, 2000-2004 1/

(In millions of Eastern Caribbean dollars; end of period)

\begin{tabular}{|c|c|c|c|c|c|}
\hline & 2000 & 2001 & 2002 & 2003 & $\begin{array}{l}\text { Prel. } \\
2004 \\
\end{array}$ \\
\hline Net international reserves & 78.2 & 82.1 & 114.9 & 118.7 & 90.8 \\
\hline ECCB's net imputed reserves & 78.2 & 82.1 & 122.8 & 128.8 & 114.1 \\
\hline Government of Dominica & $\ldots$ & $\ldots$ & -7.9 & -10.2 & -23.3 \\
\hline Net claims on commercial banks & -49.4 & -53.5 & -91.4 & -88.1 & -75.8 \\
\hline Claims & 3.9 & 0.0 & 0.0 & 0.0 & 0.0 \\
\hline Loans to banks & 0.0 & 0.0 & 0.0 & 0.0 & 0.0 \\
\hline Deposits with banks & 3.9 & 0.0 & 0.0 & 0.0 & 0.0 \\
\hline Liabilities & -53.3 & -53.5 & -91.5 & -88.1 & -75.8 \\
\hline Bankers' reserves & -40.3 & -40.3 & -74.9 & -71.4 & -60.6 \\
\hline Fixed deposits & -3.3 & -3.3 & -3.3 & -3.3 & -3.3 \\
\hline Currency & -13.0 & -13.2 & -16.5 & -16.7 & -15.2 \\
\hline Net domestic assets & 6.6 & 6.0 & 12.1 & 3.6 & 22.6 \\
\hline Net credit to central government & 10.9 & 10.2 & 21.4 & 25.8 & 34.8 \\
\hline Treasury bills & 2.5 & 2.5 & 2.5 & 2.5 & 0.6 \\
\hline Debentures & 6.5 & 6.5 & 6.5 & 6.5 & 6.5 \\
\hline Temporary advances & 0.9 & 0.7 & 0.0 & 0.0 & 0.0 \\
\hline Other claims $2 /$ & 1.3 & 0.9 & 5.4 & 7.2 & 5.1 \\
\hline Government deposits & 0.4 & 0.4 & 1.0 & 0.5 & 0.7 \\
\hline Counterpart to IMF credit & $\ldots$ & $\ldots$ & 7.9 & 10.2 & 23.3 \\
\hline Net credit to public entities & -0.9 & -0.9 & -0.9 & -1.0 & -1.0 \\
\hline Other items (net) & -3.3 & -3.3 & -8.5 & -21.2 & -11.2 \\
\hline Currency in circulation & 35.4 & 34.6 & 35.5 & 34.2 & 37.6 \\
\hline Total currency issued & 48.5 & 47.8 & 52.0 & 50.9 & 52.8 \\
\hline Currency held by banks & -13.0 & -13.2 & -16.5 & -16.7 & -15.2 \\
\hline
\end{tabular}

Sources: Eastern Caribbean Central Bank (ECCB); and Fund staff estimates.

1/ From 2002, transactions with the IMF are included as transactions with the monetary authorities.

2/ Includes balances on ECCB fiscal tranche loans to the Government of Dominica. 
Table 19. Dominica: Consolidated Balance Sheet of Commercial Banks, 2000-2004

(In millions of Eastern Caribbean dollars; end of period)

\begin{tabular}{|c|c|c|c|c|c|}
\hline & 2000 & 2001 & 2002 & 2003 & $\begin{array}{l}\text { Prel. } \\
2004 \\
\end{array}$ \\
\hline Net foreign assets & -12.9 & 14.0 & 79.0 & 170.4 & 242.9 \\
\hline Assets & 120.6 & 138.3 & 207.9 & 275.2 & 402.7 \\
\hline Foreign currency & 3.9 & 3.8 & 4.0 & 3.6 & 4.1 \\
\hline Claims on ECCB banks & 18.3 & 21.1 & 41.0 & 66.3 & 123.1 \\
\hline Claims on banks abroad & 73.0 & 84.8 & 139.2 & 115.1 & 110.9 \\
\hline Other & 25.4 & 28.6 & 23.7 & 90.2 & 164.5 \\
\hline Liabilities & -133.6 & -124.3 & -129.0 & -104.8 & -159.8 \\
\hline Balances due to ECCB banks & -44.8 & -29.6 & -30.3 & -4.0 & -11.9 \\
\hline Balances due to banks abroad & -1.6 & -0.7 & -0.9 & -1.9 & -3.2 \\
\hline Nonresident deposits & -86.7 & -93.4 & -97.1 & -98.4 & -144.0 \\
\hline Demand & -7.4 & -10.2 & -13.3 & -10.2 & -16.7 \\
\hline Savings & -66.9 & -68.2 & -70.9 & -72.6 & -87.1 \\
\hline Time & -12.3 & -14.8 & -12.6 & -13.6 & -24.8 \\
\hline Foreign currency & -0.1 & -0.2 & -0.3 & -2.0 & -15.4 \\
\hline Other & -0.5 & -0.6 & -0.7 & -0.5 & -0.7 \\
\hline Net claims on ECCB & 51.2 & 58.5 & 98.2 & 85.6 & 73.8 \\
\hline Claims on ECCB & 56.5 & 58.5 & 98.2 & 85.6 & 73.8 \\
\hline ECCB notes and coins & 13.0 & 13.2 & 16.5 & 16.7 & 15.2 \\
\hline Current deposits (including reserves) & 39.1 & 32.7 & 64.3 & 49.2 & 54.2 \\
\hline Fixed deposits & 4.4 & 12.6 & 17.4 & 19.7 & 4.4 \\
\hline Liabilities to ECCB & -5.3 & 0.0 & 0.0 & 0.0 & 0.0 \\
\hline Net domestic assets & 397.3 & 398.9 & 336.6 & 264.3 & 233.1 \\
\hline Net credit to the public sector & 65.0 & 92.1 & 53.4 & 26.3 & -10.8 \\
\hline Central and local governments & 59.5 & 82.1 & 42.8 & 30.1 & 10.9 \\
\hline Treasury bills & 16.0 & 16.0 & 16.0 & 30.8 & 15.6 \\
\hline Debentures & 26.7 & 28.7 & 26.2 & 8.0 & 26.2 \\
\hline Loans and advances & 60.1 & 65.5 & 61.7 & 52.8 & 15.9 \\
\hline Deposits & -43.4 & -28.1 & -61.1 & -61.5 & -46.9 \\
\hline Social Security Scheme & -14.3 & -10.8 & -9.0 & -21.2 & -34.8 \\
\hline Loans and advances & 0.0 & 0.0 & 0.0 & 0.0 & 0.0 \\
\hline Deposits & -14.3 & -10.8 & -9.0 & -21.2 & -34.8 \\
\hline Public enterprises & 19.8 & 20.8 & 19.6 & 17.4 & 13.1 \\
\hline Loans and advances & 27.8 & 24.3 & 23.4 & 22.2 & 21.6 \\
\hline Deposits & -8.0 & -3.5 & -3.8 & -4.8 & -8.5 \\
\hline Net credit to nonbank financial institutions & -35.8 & -37.7 & -46.6 & -81.8 & -75.9 \\
\hline Loans, advances, and investment & 3.1 & 2.9 & 2.0 & 2.4 & 2.4 \\
\hline Deposits & -38.9 & -40.5 & -48.6 & -84.2 & -78.3 \\
\hline Credit to the private sector & 454.1 & 439.6 & 433.2 & 420.6 & 444.7 \\
\hline Interbank float & 1.4 & 6.1 & -3.6 & -0.9 & -6.9 \\
\hline Other items (net) & -87.4 & -101.3 & -99.9 & -99.8 & -118.1 \\
\hline Private sector deposits & 435.6 & 471.4 & 513.7 & 520.3 & 549.8 \\
\hline Demand deposits & 54.9 & 57.0 & 71.1 & 73.7 & 73.6 \\
\hline Savings deposits & 249.0 & 259.0 & 262.2 & 279.0 & 328.5 \\
\hline Time deposits & 121.7 & 139.5 & 158.2 & 157.4 & 134.3 \\
\hline Foreign currency deposits & 10.0 & 15.9 & 22.3 & 10.2 & 13.4 \\
\hline $\begin{array}{l}\text { Memorandum item (in percent): } \\
\text { Growth in private sector deposits }\end{array}$ & 0.3 & 8.2 & 9.0 & 1.3 & 5.7 \\
\hline
\end{tabular}

Source: Eastern Caribbean Central Bank (ECCB). 
Table 20. Dominica: Sectoral Distribution of Loans and Advances

of Commercial Banks, 2000-2004

\begin{tabular}{|c|c|c|c|c|c|}
\hline & 2000 & 2001 & 2002 & 2003 & $\begin{array}{l}\text { Prel. } \\
2004\end{array}$ \\
\hline \multicolumn{6}{|c|}{ (In thousands of Eastern Caribbean dollars; end of period) } \\
\hline Total & 555,930 & 546,753 & 529,087 & 505,882 & 501,300 \\
\hline Agriculture & 11,724 & 9,754 & 9,929 & 9,276 & 9,202 \\
\hline Mining and quarrying & 6,267 & 5,264 & 6,208 & 6,954 & 6,125 \\
\hline Manufacturing & 28,162 & 19,002 & 17,761 & 17,937 & 15,606 \\
\hline Distributive trade & 77,794 & 75,732 & 75,340 & 67,315 & 79,025 \\
\hline Tourism & 26,027 & 22,793 & 21,035 & 21,032 & 17,368 \\
\hline Transportation & 20,225 & 20,545 & 19,450 & 4,565 & 4,559 \\
\hline Utilities & 40,153 & 37,147 & 36,413 & 35,314 & 37,055 \\
\hline Construction and land development & 20,010 & 17,474 & 14,714 & 16,295 & 18,555 \\
\hline Financial institutions & 16,788 & 18,542 & 1,280 & 3,689 & 4,449 \\
\hline Professional and other services & 20,739 & 19,933 & 20,542 & 18,006 & 19,562 \\
\hline Government and statutory bodies & 60,419 & 66,863 & 73,929 & 64,473 & 26,160 \\
\hline Personal & 224,961 & 230,751 & 229,951 & 237,881 & 261,125 \\
\hline Acquisition of property & 128,376 & 129,769 & 138,732 & 162,638 & 172,802 \\
\hline Durable consumer goods & 12,989 & 11,190 & 9,159 & 20,255 & 8,671 \\
\hline Other personal & 83,596 & 89,792 & 82,060 & 54,988 & 79,652 \\
\hline Other & 2,661 & 2,953 & 2,535 & 3,145 & 2,509 \\
\hline \multicolumn{6}{|c|}{ (As a percent of total) } \\
\hline Total & 100.0 & 100.0 & 100.0 & 100.0 & 100.0 \\
\hline Agriculture & 2.1 & 1.8 & 1.9 & 1.8 & 1.8 \\
\hline Mining and quarrying & 1.1 & 1.0 & 1.2 & 1.4 & 1.2 \\
\hline Manufacturing & 5.1 & 3.5 & 3.4 & 3.5 & 3.1 \\
\hline Distributive trade & 14.0 & 13.9 & 14.2 & 13.3 & 15.8 \\
\hline Tourism & 4.7 & 4.2 & 4.0 & 4.2 & 3.5 \\
\hline Transportation & 3.6 & 3.8 & 3.7 & 0.9 & 0.9 \\
\hline Utilities & 7.2 & 6.8 & 6.9 & 7.0 & 7.4 \\
\hline Construction and land development & 3.6 & 3.2 & 2.8 & 3.2 & 3.7 \\
\hline Financial institutions & 3.0 & 3.4 & 0.2 & 0.7 & 0.9 \\
\hline Professional and other services & 3.7 & 3.6 & 3.9 & 3.6 & 3.9 \\
\hline Government and statutory bodies & 10.9 & 12.2 & 14.0 & 12.7 & 5.2 \\
\hline Personal & 40.5 & 42.2 & 43.5 & 47.0 & 52.1 \\
\hline Acquisition of Property & 23.1 & 23.7 & 26.2 & 32.1 & 34.5 \\
\hline Durable Consumer Goods & 2.3 & 2.0 & 1.7 & 4.0 & 1.7 \\
\hline Other Personal & 15.0 & 16.4 & 15.5 & 10.9 & 15.9 \\
\hline Other & 0.5 & 0.5 & 0.5 & 0.6 & 0.5 \\
\hline
\end{tabular}

Source: Eastern Caribbean Central Bank (ECCB). 
Table 21. Dominica: Interest Rates, 2000-2004 1/

(In percent; end of period)

\begin{tabular}{|c|c|c|c|c|c|}
\hline & 2000 & 2001 & 2002 & 2003 & 2004 \\
\hline \multicolumn{6}{|c|}{ COMMERCIAL BANK LENDING RATES } \\
\hline Banks' prime loan rate (maximum) & 10.50 & 10.50 & 10.50 & 10.50 & 10.00 \\
\hline Weighted average lending rate & 11.71 & 11.02 & 11.03 & 11.78 & 9.78 \\
\hline Local currency & $\ldots$ & $\ldots$ & $\ldots$ & 11.94 & 9.82 \\
\hline Foreign currency & $\ldots$ & $\ldots$ & $\ldots$ & 5.78 & 4.46 \\
\hline \multicolumn{6}{|c|}{ COMMERCIAL BANK DEPOSIT RATES } \\
\hline Total deposits & 3.95 & 3.98 & 3.62 & 3.50 & 3.04 \\
\hline Local currency & $\ldots$ & $\ldots$ & $\ldots$ & 3.58 & 3.19 \\
\hline Foreign currency & $\ldots$ & $\ldots$ & $\ldots$ & 1.01 & 0.21 \\
\hline Demand deposits & $\ldots$ & $\ldots$ & $\ldots$ & 1.35 & 0.83 \\
\hline Local currency & $\ldots$ & $\ldots$ & $\ldots$ & 1.44 & 1.00 \\
\hline Foreign currency & $\ldots$ & $\ldots$ & $\ldots$ & 1.01 & 0.13 \\
\hline Savings deposits & $\ldots$ & $\ldots$ & $\ldots$ & 3.19 & 3.34 \\
\hline Local currency & $\ldots$ & $\ldots$ & $\ldots$ & 3.19 & 3.34 \\
\hline Foreign currency & $\ldots$ & $\ldots$ & $\ldots$ & 3.00 & 1.68 \\
\hline Time deposits & $\ldots$ & $\ldots$ & $\ldots$ & 6.00 & 4.60 \\
\hline Local currency & $\ldots$ & $\ldots$ & $\ldots$ & 6.00 & 4.68 \\
\hline Foreign currency & $\ldots$ & $\ldots$ & $\ldots$ & 0.00 & 0.90 \\
\hline \multicolumn{6}{|l|}{ INTEREST RATE SPREADS } \\
\hline Overall spread & 7.76 & 7.04 & 7.40 & 8.28 & 6.74 \\
\hline Local currency & $\ldots$ & $\ldots$ & $\ldots$ & 8.36 & 6.63 \\
\hline Foreign currency & $\ldots$ & $\ldots$ & $\ldots$ & 4.76 & 4.26 \\
\hline \multicolumn{6}{|l|}{ OTHER INTEREST RATES } \\
\hline Interbank market rate & 5.25 & 6.79 & 6.18 & 5.30 & 4.57 \\
\hline 3-month treasury bill rate & 6.40 & 6.40 & 6.40 & 6.40 & 6.40 \\
\hline ECCB's discount rate & 8.00 & 7.00 & 7.00 & 6.50 & 6.50 \\
\hline
\end{tabular}

Source: Eastern Caribbean Central Bank (ECCB).

1/ Improved reporting requirements were introduced from June 2003, which provide information on deposit and lending rates by currency and an accurate calcuation of the weighted average rates. There is therefore a break in the data series in 2003. 
Table 22. Dominica: Financial Accounts of the Dominica Agricultural and Development Bank, 2000-2004

(In millions of Eastern Caribbean dollars)

\begin{tabular}{|c|c|c|c|c|c|}
\hline & 2000 & 2001 & 2002 & 2003 & 2004 \\
\hline \multicolumn{6}{|c|}{ Balance Sheet, as at June 30} \\
\hline Assets & 119.0 & 118.4 & 119.8 & 120.1 & 126.7 \\
\hline Cash and short-term deposits & 7.6 & 3.9 & 5.6 & 2.3 & 8.3 \\
\hline Accounts receivable & 3.5 & 3.5 & 3.2 & 2.4 & 2.6 \\
\hline Loans outstanding & 98.2 & 101.4 & 102.4 & 106.5 & 106.7 \\
\hline Agriculture & 12.6 & 12.4 & 11.7 & 11.0 & 10.6 \\
\hline Industry & 17.3 & 17.7 & 15.4 & 17.5 & 19.9 \\
\hline Tourism & 15.2 & 14.8 & 20.1 & 19.8 & 19.7 \\
\hline Mortgage & 31.3 & 32.7 & 28.0 & 30.5 & 28.0 \\
\hline Student loans & 16.1 & 18.9 & 22.8 & 27.2 & 30.4 \\
\hline Other & 11.5 & 11.5 & 12.1 & 11.6 & 10.3 \\
\hline Less: Provision for doubtful loans & 5.7 & 6.5 & 7.7 & 11.0 & 12.3 \\
\hline Investments & 3.6 & 3.6 & 2.5 & 3.4 & 3.9 \\
\hline Government debentures and bills & 2.2 & 2.2 & 2.2 & 2.2 & 2.6 \\
\hline Other & 1.4 & 1.4 & 0.3 & 1.2 & 1.3 \\
\hline Agency assets & 0.1 & 0.2 & 0.2 & $\ldots$ & $\ldots$ \\
\hline Fixed assets & 6.1 & 5.9 & 5.9 & 5.5 & 5.2 \\
\hline Liabilities & 119.0 & 118.4 & 119.8 & 120.1 & 126.7 \\
\hline Long-term liabilities & 80.5 & 78.7 & 82.3 & 85.5 & 89.0 \\
\hline Caribbean Development Bank & 41.7 & 42.0 & 45.6 & 48.9 & 54.7 \\
\hline Dominica Social Security & 19.1 & 18.3 & 18.4 & 19.2 & 18.0 \\
\hline European Investment Bank & 5.2 & 5.9 & 7.9 & 9.2 & 9.8 \\
\hline Taiwan, Province of China & 4.6 & 4.4 & 4.2 & 4.0 & 3.9 \\
\hline France & 3.9 & 3.3 & 2.8 & 2.0 & 1.4 \\
\hline IFAD & 2.6 & 2.2 & 1.9 & 1.5 & 1.2 \\
\hline $\begin{array}{l}\text { Commonwealth Development Corporation } \\
\text { Other }\end{array}$ & 3.6 & 2.5 & 1.5 & 0.5 & 0.0 \\
\hline Interest bearing deposits & 7.1 & 7.0 & 5.4 & 5.7 & 5.8 \\
\hline Agency liabilities & $\ldots$ & $\ldots$ & $\ldots$ & 0.2 & 1.0 \\
\hline Accounts payable and accrued liabilities & 2.4 & 2.3 & 1.9 & 2.5 & 3.7 \\
\hline Dividend payable & 0.3 & 0.3 & 0.3 & 0.4 & 0.3 \\
\hline Shareholders' equity and deferred grants & 28.8 & 30.1 & 29.9 & 26.0 & 27.0 \\
\hline
\end{tabular}

Statement of Earnings, for year ending June 30

Total revenue

Interest on loans and advances

Interest on fixed deposits

Other

Total expenditure

Interest on long-term loans

Personnel expenditure

Other expenses

Depreciation

Net surplus/deficit

Provision for loan loss and other losses

Net earnings/loss

\section{4}

8.6

0.5

0.4

7.3

3.7

2.1

1.3

0.3

2.2

$-0.7$

1.5
9.7

8.8

0.4

0.4

7.6

3.9

2.0

1.5

0.2

2.0

$-0.5$

1.6
10.1

9.2

0.4

0.5

\section{3}

3.9

2.3

1.6

0.6

1.8

$-0.9$

0.9
9.4

8.3

0.3

0.8

8.1

4.0

2.3

1.3

0.4

1.3

$-3.1$

$-1.8$

Memorandum items:

Nonperforming loans/total loans (in percent)

$\cdots$ 
Table 23. Dominica: Consolidated Balance Sheet of the Major Credit Unions, 2000-2004

\begin{tabular}{|c|c|c|c|c|c|}
\hline & 2000 & 2001 & 2002 & 2003 & $\begin{array}{c}\text { Prel. } \\
2004 \\
\end{array}$ \\
\hline \multicolumn{6}{|c|}{ (In millions of Eastern Caribbean dollars; end of period) } \\
\hline Assets & 238.7 & 255.1 & 273.0 & 290.2 & 316.2 \\
\hline Cash balance & 8.0 & 10.4 & 13.4 & 11.4 & 12.9 \\
\hline Investments & 24.6 & 25.4 & 32.5 & 40.1 & 52.7 \\
\hline Loans outstanding & 186.6 & 197.6 & 206.9 & 218.3 & 229.0 \\
\hline Agriculture & 2.5 & 2.1 & 2.2 & 2.1 & 2.3 \\
\hline Business & 6.6 & 6.9 & 6.2 & 6.2 & 8.2 \\
\hline Mortgages & 85.6 & 95.7 & 102.2 & 109.4 & 110.7 \\
\hline Educational & 1.8 & 2.3 & 3.3 & 3.8 & 4.6 \\
\hline Personal & 90.1 & 90.5 & 93.0 & 96.7 & 103.2 \\
\hline Fixed assets & 15.0 & 15.9 & 15.2 & 15.2 & 16.1 \\
\hline Other assets & 4.5 & 5.8 & 5.0 & 5.2 & 5.6 \\
\hline Liabilities & 238.7 & 255.1 & 273.0 & 290.2 & 316.2 \\
\hline Share capital & 120.3 & 121.8 & 123.7 & 132.4 & 111.5 \\
\hline Deposits & 76.6 & 86.4 & 99.9 & 108.8 & 142.4 \\
\hline Savings & 10.9 & 9.9 & 11.3 & 13.2 & 42.7 \\
\hline Demand & 3.5 & 4.8 & 5.0 & 4.5 & 6.8 \\
\hline Time & 60.0 & 67.4 & 78.2 & 83.7 & 78.9 \\
\hline Other & 2.1 & 4.3 & 5.3 & 7.3 & 14.0 \\
\hline Loans received & 12.4 & 11.5 & 11.2 & 9.6 & 7.1 \\
\hline Capital & 23.1 & 27.7 & 27.2 & 32.2 & 35.1 \\
\hline Other liabilities & 6.3 & 7.7 & 11.1 & 7.2 & 20.2 \\
\hline \multicolumn{6}{|c|}{ (in millions of Eastern Caribbean dollars, unless otherwise indicated) } \\
\hline \multicolumn{6}{|l|}{ Memorandum item: } \\
\hline Total Income & 25.1 & 29.6 & 31.1 & 31.6 & 22.6 \\
\hline Interest & 24.3 & 28.7 & 30.1 & 30.6 & 21.5 \\
\hline Other & 0.8 & 0.9 & 1.0 & 1.0 & 1.1 \\
\hline Total expenditure & 18.1 & 19.4 & 24.1 & 21.9 & 14.7 \\
\hline Interest & 8.0 & 4.7 & 8.3 & 7.4 & 4.4 \\
\hline Personnel expenditure & 5.0 & 5.9 & 6.1 & 6.1 & 4.3 \\
\hline Other & 5.1 & 8.7 & 9.7 & 8.4 & 5.9 \\
\hline Gross surplus/deficit & 7.0 & 10.3 & 7.0 & 9.7 & 8.0 \\
\hline Nonperforming loans & 21.4 & 24.3 & 18.2 & 19.5 & 25.2 \\
\hline Nonperforming loans/total loans (percent) & 11.5 & 12.3 & 8.8 & 8.9 & 11.0 \\
\hline Credit Union membership (number) & 65,159 & 66,033 & 72,547 & 71,474 & 71,060 \\
\hline Reporting credit unions (number) & 10 & 9 & 5 & 8 & 8 \\
\hline Credit unions not reporting (number) & 9 & 8 & 12 & 9 & 9 \\
\hline
\end{tabular}

Source: Eastern Caribbean Central Bank (ECCB). 
Table 24. Dominica: Balance of Payments, 2000-2004

\begin{tabular}{|c|c|c|c|c|c|}
\hline & 2000 & 2001 & 2002 & 2003 & $\begin{array}{l}\text { Prel. } \\
2004\end{array}$ \\
\hline \multicolumn{6}{|c|}{ (In millions of U.S. dollars) } \\
\hline Current account balance & -53.1 & -48.9 & -34.7 & -33.6 & -46.7 \\
\hline Trade balance & -75.6 & -71.3 & -58.7 & -70.8 & -84.6 \\
\hline Exports (f.o.b.) & 54.7 & 44.4 & 43.6 & 41.0 & 42.2 \\
\hline Of which: bananas & 12.0 & 8.2 & 8.1 & 5.9 & 7.2 \\
\hline Imports (f.o.b.) & 130.4 & 115.7 & 102.4 & 111.8 & 126.9 \\
\hline Services balance & 37.1 & 25.6 & 26.0 & 32.8 & 40.0 \\
\hline Exports of services & 89.7 & 75.6 & 79.7 & 77.3 & 86.3 \\
\hline Travel & 48.2 & 46.4 & 45.7 & 52.3 & 60.1 \\
\hline Other & 41.6 & 29.2 & 34.0 & 25.0 & 26.2 \\
\hline Imports of services & 52.7 & 50.0 & 53.7 & 44.6 & 46.3 \\
\hline Net Income & -32.5 & -20.6 & -18.4 & -12.1 & -21.8 \\
\hline Interest payments (public sector) & -6.2 & -7.7 & -8.8 & -8.0 & -8.6 \\
\hline Other & -26.3 & -12.9 & -9.6 & -4.1 & -13.2 \\
\hline Net current transfers & 18.1 & 17.5 & 16.4 & 16.6 & 19.8 \\
\hline Private & 10.6 & 11.5 & 12.6 & 12.4 & 18.4 \\
\hline Public & 7.4 & 5.9 & 3.8 & 4.2 & 1.4 \\
\hline Capital and financial account & 57.4 & 40.9 & 32.1 & 22.4 & $\mathbf{2 3 . 0}$ \\
\hline Capital account & 10.9 & 18.0 & 20.5 & 18.8 & 26.8 \\
\hline Public capital transfers & 9.6 & 15.3 & 17.7 & 15.9 & 23.8 \\
\hline Private capital transfers & 2.7 & 2.7 & 2.8 & 2.9 & 3.0 \\
\hline Financial account & 46.5 & 22.9 & 11.6 & 3.7 & -3.8 \\
\hline Public sector & 22.0 & 20.1 & 25.8 & 8.9 & 0.2 \\
\hline Budgetary flows & 23.2 & 21.7 & 25.0 & 8.9 & 0.2 \\
\hline Disbursements & 27.7 & 27.1 & 30.7 & 24.0 & 18.3 \\
\hline Repayments & 4.5 & 5.4 & 5.7 & 15.1 & 18.1 \\
\hline Nonbudgetary flows (net) & -1.2 & -1.6 & 0.8 & 0.0 & 0.0 \\
\hline Private sector & 24.5 & 2.8 & -14.1 & -5.2 & -4.0 \\
\hline Direct investment & 10.8 & 11.9 & 11.4 & 19.8 & 18.1 \\
\hline Commercial banks & 19.7 & -10.0 & -24.1 & -33.9 & -26.9 \\
\hline Other private flows & -5.9 & 0.9 & -1.5 & 8.8 & 4.8 \\
\hline Errors and omissions & -6.9 & 9.4 & 14.7 & 12.5 & -2.1 \\
\hline Overall balance & -2.5 & 1.5 & 12.1 & 1.4 & -25.7 \\
\hline Overall financing & 2.5 & -1.5 & -12.1 & -1.4 & 25.7 \\
\hline Net international reserves & 2.5 & -1.5 & -12.1 & -1.4 & 10.3 \\
\hline Gross reserves (increase $=-$ ) & 2.5 & -1.5 & -15.1 & -6.2 & 9.4 \\
\hline IMF reserve liabilities (purchase $=+$ ) & 0.0 & 0.0 & 3.0 & 4.8 & 0.9 \\
\hline Exceptional financing & 0.0 & 0.0 & 0.0 & 0.0 & 15.4 \\
\hline \multicolumn{6}{|c|}{ (As a percentage of GDP) } \\
\hline \multicolumn{6}{|l|}{ Memorandum items: } \\
\hline Trade balance & -28.1 & -27.2 & -23.3 & -27.4 & -31.2 \\
\hline Current account balance & -19.7 & -18.7 & -13.8 & -13.0 & -17.2 \\
\hline Current account balance including & & & & & \\
\hline net capital transfers & -15.7 & -11.8 & -5.6 & -5.7 & -7.3 \\
\hline Capital and financial account & 21.4 & 15.6 & 12.7 & 8.7 & 5.9 \\
\hline \multicolumn{6}{|l|}{ External public debt service (as a percent } \\
\hline of exports of goods and nonfactor services): & 7.4 & 10.9 & 11.8 & 19.5 & 20.8 \\
\hline Amortization & 3.1 & 4.5 & 4.6 & 12.8 & 14.1 \\
\hline Interest & 4.3 & 6.4 & 7.1 & 6.8 & 6.7 \\
\hline
\end{tabular}

Sources: Dominican authorities; Eastern Caribbean Central Bank (ECCB); and Fund staff estimates. 
Table 25. Dominica: Exports by Major Commodities, 2000-2004

\begin{tabular}{|c|c|c|c|c|c|}
\hline & 2000 & 2001 & 2002 & 2003 & $\begin{array}{l}\text { Prel. } \\
2004 \\
\end{array}$ \\
\hline \multicolumn{6}{|c|}{$\begin{array}{l}\text { (Value in millions of U.S. dollars; volume in thousands of metric tons, unless otherwise } \\
\text { specified; and unit price in U.S. dollars per metric ton) }\end{array}$} \\
\hline Value of exports of goods & 54.7 & 44.4 & 43.6 & 41.0 & 42.2 \\
\hline Domestic merchandise exports & 50.9 & 41.4 & 39.5 & 38.6 & 39.4 \\
\hline Merchandise re-exports & 2.6 & 2.0 & 3.2 & 1.4 & 1.7 \\
\hline Stores and bunkers & 1.2 & 1.0 & 0.9 & 1.0 & 1.0 \\
\hline Agricultural exports & 18.1 & 14.3 & 14.2 & 11.5 & 13.4 \\
\hline \multicolumn{6}{|l|}{ Bananas } \\
\hline Value & 12.0 & 8.2 & 8.1 & 5.9 & 7.2 \\
\hline Volume & 28.8 & 19.1 & 18.4 & 12.0 & 14.5 \\
\hline Unit value & 417.6 & 431.9 & 440.2 & 491.7 & 496.6 \\
\hline \multicolumn{6}{|l|}{ Other agricultural exports } \\
\hline Value & 6.1 & 6.1 & 6.0 & 5.6 & 6.2 \\
\hline Manufactured exports & 32.8 & 27.3 & 25.2 & 27.1 & 26.1 \\
\hline \multicolumn{6}{|l|}{ Soap } \\
\hline Value & 13.5 & 11.4 & 10.0 & 10.8 & 11.1 \\
\hline Volume & 11.3 & 10.2 & 8.6 & 9.1 & 8.8 \\
\hline Unit value & $1,189.6$ & $1,117.4$ & $1,162.8$ & $1,186.8$ & $1,261.4$ \\
\hline \multicolumn{6}{|l|}{ Other manufactured exports } \\
\hline Value & 19.3 & 15.9 & 15.2 & 16.4 & 15.0 \\
\hline \multicolumn{6}{|c|}{ (In percent of total exports) } \\
\hline Agricultural exports & 33.1 & 32.3 & 32.5 & 28.0 & 31.8 \\
\hline Bananas & 22.0 & 18.5 & 18.7 & 14.4 & 17.2 \\
\hline Other agricultural exports & 11.1 & 13.8 & 13.8 & 13.6 & 14.6 \\
\hline Manufactured exports & 59.9 & 61.6 & $\mathbf{5 7 . 8}$ & 66.2 & 61.9 \\
\hline Soap & 24.6 & 25.7 & 22.9 & 26.3 & 26.4 \\
\hline Other manufactured exports & 35.3 & 35.9 & 34.9 & 39.9 & 35.5 \\
\hline
\end{tabular}

Sources: Central Statistical Office, Ministry of Finance; and Fund staff estimates. 
Table 26. Dominica: Imports by Product Type, 2000-2004 1/

(In millions of U.S. dollars)

\begin{tabular}{|c|c|c|c|c|c|}
\hline & 2000 & 2001 & 2002 & 2003 & $\begin{array}{l}\text { Prel. } \\
2004 \\
\end{array}$ \\
\hline Total & 147.8 & 130.6 & 115.6 & 127.1 & 144.2 \\
\hline Food products and tobacco & 29.9 & 26.0 & 25.4 & 25.9 & 27.6 \\
\hline Meats and meat preparations & 5.5 & 4.8 & 4.8 & 4.5 & 5.4 \\
\hline Dairy products and eggs & 4.2 & 3.7 & 3.3 & 3.7 & 3.8 \\
\hline Fish & 1.6 & 1.3 & 1.4 & 1.3 & 1.5 \\
\hline Cereals and cereal preparations & 5.2 & 4.6 & 4.4 & 4.4 & 4.5 \\
\hline Vegetables and fruits & 2.1 & 2.0 & 2.0 & 2.1 & 2.1 \\
\hline Others (including beverages and tobacco) & 11.3 & 9.6 & 9.5 & 9.9 & 10.3 \\
\hline Mineral fuels & 14.5 & 12.9 & 10.9 & 13.7 & 16.0 \\
\hline Miscellaneous oils and chemicals & 9.6 & 9.7 & 8.1 & 9.8 & 10.4 \\
\hline Animal and vegetable oils & 2.5 & 3.4 & 2.4 & 4.1 & 3.9 \\
\hline Dyeing and tanning materials & 1.6 & 1.3 & 1.4 & 1.4 & 1.5 \\
\hline Essential oils and resinoids & 4.4 & 4.3 & 3.7 & 3.5 & 4.0 \\
\hline Fertilizers & 1.0 & 0.7 & 0.6 & 0.8 & 1.0 \\
\hline Miscellaneous manufactures & 26.9 & 23.5 & 23.8 & 24.4 & 27.3 \\
\hline Rubber products & 1.9 & 1.6 & 1.4 & 1.6 & 2.0 \\
\hline Cork and wood products & 2.4 & 2.1 & 1.3 & 1.2 & 2.1 \\
\hline Paper products & 5.0 & 4.4 & 5.7 & 5.0 & 5.6 \\
\hline Printed matter & 1.1 & 1.0 & 1.0 & 1.0 & 1.0 \\
\hline Textile yarn and fabrics & 1.4 & 1.2 & 1.2 & 1.2 & 1.6 \\
\hline Footwear & 0.8 & 0.7 & 0.8 & 0.8 & 0.9 \\
\hline Metal products & 6.6 & 5.8 & 4.9 & 6.1 & 6.2 \\
\hline Furniture & 1.7 & 1.5 & 1.8 & 1.8 & 2.1 \\
\hline Travel goods & 0.2 & 0.2 & 0.2 & 0.2 & 0.2 \\
\hline Nonmetal mineral manufactures & 4.2 & 3.7 & 3.2 & 3.8 & 3.7 \\
\hline Medical and pharmaceutical products & 1.6 & 1.4 & 2.3 & 1.7 & 1.9 \\
\hline Miscellaneous primary products & 6.2 & 5.4 & 3.1 & 3.7 & 5.4 \\
\hline Cork and wood & 2.9 & 2.5 & 1.3 & 1.6 & 2.2 \\
\hline Iron and steel & 3.3 & 2.9 & 1.8 & 2.1 & 3.2 \\
\hline Machinery and equipment & 38.8 & 33.9 & 25.5 & 28.5 & 36.2 \\
\hline Power generation equipment & 2.5 & 2.2 & 1.7 & 1.4 & 1.6 \\
\hline Specialized industrial machines & 4.9 & 4.2 & 1.1 & 2.5 & 2.7 \\
\hline General industrial machinery & 3.8 & 3.3 & 2.9 & 2.4 & 3.2 \\
\hline Office machines and computers & 4.2 & 3.7 & 2.9 & 2.8 & 4.3 \\
\hline Telecommunications and sound equipment & 5.7 & 5.0 & 4.6 & 7.2 & 10.5 \\
\hline Electrical machinery & 5.1 & 4.5 & 4.5 & 4.7 & 4.8 \\
\hline Vehicles & 12.4 & 10.8 & 6.8 & 6.9 & 8.6 \\
\hline Other transport equipment & 0.2 & 0.2 & 1.0 & 0.6 & 0.5 \\
\hline Other & 22.0 & 19.2 & 18.2 & 19.0 & 19.3 \\
\hline Oil seeds and oleagenous fruits & 0.0 & 0.0 & 0.0 & 0.0 & 0.0 \\
\hline Textile fabrics (other than wool tops and combed wool) & 0.0 & 0.0 & 1.3 & 0.0 & 0.0 \\
\hline Crude fertilizers & 0.2 & 0.2 & 0.3 & 0.2 & 0.2 \\
\hline Crude animals and vegetable materials & 0.4 & 0.4 & 0.3 & 0.3 & 0.4 \\
\hline Organic chemicals & 1.2 & 1.0 & 0.9 & 1.1 & 1.0 \\
\hline Inorganic chemicals & 2.0 & 1.8 & 1.8 & 1.9 & 2.0 \\
\hline Plastics in primary forms & 1.0 & 0.9 & 0.8 & 0.9 & 0.9 \\
\hline Plastics in nonprimary forms & 2.4 & 2.1 & 1.0 & 1.0 & 1.3 \\
\hline Chemical materials and products & 3.0 & 2.6 & 2.2 & 2.1 & 2.3 \\
\hline Prefabricated buildings, etc. & 0.7 & 0.6 & 0.6 & 1.6 & 0.9 \\
\hline Professional, scientific instruments & 1.1 & 1.0 & 1.4 & 2.0 & 1.6 \\
\hline Photographic apparatus & 0.7 & 0.6 & 0.6 & 0.7 & 0.9 \\
\hline Miscellaneous manufactured articles & 9.2 & 8.0 & 7.0 & 7.2 & 7.8 \\
\hline
\end{tabular}

Source: Central Statistical Office, Ministry of Finance.

1/ On c.i.f. basis. 
Table 27. Dominica: Direction of Trade, 2000-2004

\begin{tabular}{|c|c|c|c|c|c|}
\hline & 2000 & 2001 & 2002 & 2003 & $\begin{array}{l}\text { Prel. } \\
2004\end{array}$ \\
\hline \multicolumn{6}{|c|}{ (In millions of U.S. dollars) } \\
\hline Domestic merchandise exports 1 / & 53.6 & 43.4 & 42.7 & 40.0 & 41.2 \\
\hline United Kingdom & 12.5 & 9.5 & 9.4 & 6.2 & 7.4 \\
\hline United States (including Puerto Rico) & 2.3 & 2.0 & 3.4 & 2.2 & 1.6 \\
\hline Guadeloupe & 2.8 & 2.8 & 2.4 & 3.2 & 3.7 \\
\hline CARICOM countries & 28.1 & 27.6 & 23.7 & 25.3 & 25.2 \\
\hline Other & 7.9 & 1.5 & 3.8 & 3.1 & 3.2 \\
\hline Total merchandise imports & 147.8 & 130.6 & 115.6 & 127.1 & 144.2 \\
\hline United Kingdom & 11.3 & 13.2 & 9.9 & 9.7 & 9.6 \\
\hline United States (including Puerto Rico) & 38.4 & 47.2 & 42.2 & 46.7 & 52.7 \\
\hline Canada & 6.2 & 3.8 & 3.1 & 4.4 & 4.0 \\
\hline France & 1.3 & 0.9 & 1.2 & 1.7 & 5.1 \\
\hline Japan & 9.2 & 8.3 & 4.7 & 5.0 & 6.8 \\
\hline The Netherlands & 3.2 & 2.3 & 2.2 & 2.1 & 2.0 \\
\hline Guadeloupe & 1.3 & 1.3 & 1.1 & 1.5 & 1.9 \\
\hline CARICOM countries & 39.4 & 36.3 & 35.2 & 38.7 & 42.9 \\
\hline Other & 37.5 & 17.3 & 16.0 & 17.3 & 19.2 \\
\hline \multicolumn{6}{|c|}{ (In percent of total) } \\
\hline Domestic merchandise exports 1 / & 100.0 & 100.0 & 100.0 & 100.0 & 100.0 \\
\hline United Kingdom & 23.3 & 21.8 & 22.0 & 15.5 & 18.0 \\
\hline United States (including Puerto Rico) & 4.3 & 4.7 & 8.0 & 5.5 & 3.9 \\
\hline Guadeloupe & 5.2 & 6.5 & 5.6 & 8.0 & 9.0 \\
\hline CARICOM countries & 52.4 & 63.5 & 55.6 & 63.3 & 61.2 \\
\hline Other & 14.7 & 3.5 & 8.8 & 7.8 & 7.9 \\
\hline Total merchandise imports & 100.0 & 100.0 & 100.0 & 100.0 & 100.0 \\
\hline United Kingdom & 7.6 & 10.1 & 8.6 & 7.6 & 6.6 \\
\hline United States (including Puerto Rico) & 26.0 & 36.1 & 36.5 & 36.7 & 36.5 \\
\hline Canada & 4.2 & 2.9 & 2.7 & 3.5 & 2.7 \\
\hline France & 0.9 & 0.7 & 1.0 & 1.3 & 3.6 \\
\hline Japan & 6.2 & 6.4 & 4.1 & 3.9 & 4.7 \\
\hline The Netherlands & 2.2 & 1.8 & 1.9 & 1.6 & 1.4 \\
\hline Guadeloupe & 0.9 & 1.0 & 1.0 & 1.1 & 1.3 \\
\hline CARICOM countries & 26.7 & 27.8 & 30.4 & 30.4 & 29.7 \\
\hline Other & 25.4 & 13.3 & 13.8 & 13.6 & 13.3 \\
\hline
\end{tabular}

Sources: IMF Direction of Trade Statistics; and Fund staff estimates.

1/ Includes re-exports. 
Table 28. Dominica: Terms of Trade, 2000-2004

\begin{tabular}{|c|c|c|c|c|c|}
\hline & 2000 & 2001 & 2002 & 2003 & $\begin{array}{c}\text { Prel. } \\
2004\end{array}$ \\
\hline \multicolumn{6}{|c|}{$(1995=100)$} \\
\hline \multicolumn{6}{|l|}{ Exports of goods } \\
\hline Value & 118.8 & 96.3 & 94.6 & 88.9 & 91.6 \\
\hline Volume & 133.6 & 107.6 & 105.2 & 93.0 & $\ldots$ \\
\hline Unit value & 88.9 & 89.5 & 89.9 & 95.6 & $\ldots$ \\
\hline \multicolumn{6}{|l|}{ Imports of goods } \\
\hline Value & 131.6 & 116.7 & 103.3 & 112.9 & 128.1 \\
\hline Volume & 125.7 & 117.2 & 108.3 & 118.1 & $\ldots$ \\
\hline Unit value & 104.7 & 99.6 & 95.4 & 95.6 & $\ldots$ \\
\hline Merchandise terms of trade & 84.9 & 89.9 & 94.2 & 99.9 & $\ldots$ \\
\hline \multicolumn{6}{|c|}{ (Annual percentage change) } \\
\hline \multicolumn{6}{|l|}{ Exports } \\
\hline Value & -2.3 & -18.9 & -1.8 & -6.0 & 3.0 \\
\hline Volume & 10.6 & -19.4 & -2.2 & -11.6 & $\ldots$ \\
\hline Unit value & -11.6 & 0.7 & 0.4 & 6.3 & $\ldots$ \\
\hline \multicolumn{6}{|l|}{ Imports } \\
\hline Value & 7.3 & -11.3 & -11.5 & 9.3 & 13.5 \\
\hline Volume & 1.6 & -6.8 & -7.6 & 9.1 & $\ldots$ \\
\hline Unit value & 5.5 & -4.9 & -4.2 & 0.2 & $\ldots$ \\
\hline Merchandise terms of trade & -16.3 & 5.8 & 4.9 & 6.1 & $\ldots$ \\
\hline
\end{tabular}

Sources: Central Statistical Office, Ministry of Finance; and Fund staff estimates. 
Table 29. Dominica: Tourism Statistics, 2000-2004

\begin{tabular}{|c|c|c|c|c|c|}
\hline & 2000 & 2001 & 2002 & 2003 & $\begin{array}{l}\text { Prel. } \\
2004\end{array}$ \\
\hline \multicolumn{6}{|l|}{ Arrivals } \\
\hline Total & 305,873 & 272,908 & 200,893 & 250,802 & 462,529 \\
\hline Excursionists & 1,890 & 1,681 & 1,693 & 3,928 & 2,577 \\
\hline Cruiseship & 239,544 & 207,627 & 136,859 & 177,044 & 383,614 \\
\hline Stayover & 64,439 & 63,600 & 62,341 & 69,830 & 76,338 \\
\hline Hotel holiday & 15,247 & 15,049 & 13,901 & 13,711 & 14,993 \\
\hline Hotel business & 9,452 & 9,329 & 8,624 & 8,369 & 8,689 \\
\hline Private homes & 39,000 & 38,493 & 38,935 & 46,476 & 51,509 \\
\hline Students & 740 & 730 & 881 & 1,274 & 1,147 \\
\hline \multicolumn{6}{|c|}{ Average length of stay $1 /$} \\
\hline Excursionists & 1.0 & 1.0 & 1.0 & 1.0 & 1.0 \\
\hline Cruiseship & 1.0 & 1.0 & 1.0 & 1.0 & 1.0 \\
\hline \multicolumn{6}{|l|}{ Stayover } \\
\hline Hotel holiday & 9.1 & 9.1 & 9.1 & 9.1 & 9.1 \\
\hline Hotel business & 11.0 & 11.0 & 11.0 & 11.0 & 11.0 \\
\hline Private homes & 14.8 & 14.8 & 14.8 & 14.8 & 14.8 \\
\hline Students & 315.0 & 315.0 & 320.0 & 320.0 & 320.0 \\
\hline \multicolumn{6}{|c|}{ Average daily expenditure (EC\$) 1/ } \\
\hline Excursionists & 179.6 & 183.2 & 182.8 & 185.4 & 190.0 \\
\hline Cruiseship & 73.5 & 75.0 & 74.8 & 75.9 & 77.7 \\
\hline \multicolumn{6}{|l|}{ Stayover } \\
\hline Hotel holiday & 244.9 & 249.8 & 249.1 & 252.7 & 259.0 \\
\hline Hotel business & 304.0 & 310.1 & 309.4 & 313.8 & 321.5 \\
\hline Private homes & 37.7 & 38.4 & 38.4 & 38.9 & 39.9 \\
\hline Students & 95.1 & 97.0 & 96.8 & 98.2 & 100.6 \\
\hline \multicolumn{6}{|c|}{ Total receipts (EC\$ million) } \\
\hline Total & 127.5 & 126.1 & 120.8 & 141.4 & 163.7 \\
\hline Excursionists & 0.3 & 0.3 & 0.3 & 0.7 & 0.5 \\
\hline Cruiseship & 17.6 & 15.6 & 10.2 & 13.4 & 29.8 \\
\hline Stayover & 109.5 & 110.2 & 110.3 & 127.2 & 133.4 \\
\hline Hotel holiday & 34.0 & 34.2 & 31.5 & 31.5 & 35.3 \\
\hline Hotel business & 31.6 & 31.8 & 29.3 & 28.9 & 30.7 \\
\hline Private homes & 21.8 & 21.9 & 22.1 & 26.8 & 30.4 \\
\hline Students & 22.2 & 22.3 & 27.3 & 40.0 & 36.9 \\
\hline
\end{tabular}

Sources: Central Statistical Office, Ministry of Finance; Eastern Caribbean Central Bank (ECCB); Ross University; and Fund staff estimates.

1/ Data for students are provided by Ross University. Data for other categories are based on a 1991 survey, but average daily expenditures are adjusted annually by the food and beverage price index. 
Table 30. Dominica: Effective Exchange Rate Indices, 2000-2004 1/

\begin{tabular}{|c|c|c|c|c|}
\hline & & $\begin{array}{r}\text { Real } \\
\text { Effective } \\
\text { Rate } \\
\end{array}$ & $\begin{array}{r}\text { Nominal } \\
\text { Effective } \\
\text { Rate } \\
\end{array}$ & $\begin{array}{r}\text { Relative } \\
\text { Price } \\
\end{array}$ \\
\hline \multicolumn{5}{|c|}{ (Index numbers: $1990=100$ ) } \\
\hline & & (Quarterly ave & & \\
\hline \multirow[t]{4}{*}{2000} & I & 106.1 & 138.3 & 76.7 \\
\hline & II & 108.6 & 140.9 & 77.1 \\
\hline & III & 109.4 & 142.8 & 76.6 \\
\hline & IV & 111.1 & 146.0 & 76.1 \\
\hline \multirow[t]{4}{*}{2001} & $\mathrm{I}$ & 111.6 & 146.1 & 76.4 \\
\hline & II & 114.3 & 149.8 & 76.3 \\
\hline & III & 113.0 & 149.0 & 75.9 \\
\hline & IV & 113.0 & 149.2 & 75.7 \\
\hline \multirow[t]{4}{*}{2002} & $\mathrm{I}$ & 114.2 & 151.4 & 75.4 \\
\hline & II & 111.3 & 148.0 & 75.2 \\
\hline & III & 107.1 & 143.5 & 74.6 \\
\hline & IV & 107.1 & 144.0 & 74.4 \\
\hline \multirow[t]{4}{*}{2003} & I & 103.8 & 140.3 & 74.0 \\
\hline & II & 101.4 & 137.8 & 73.6 \\
\hline & III & 103.6 & 137.6 & 75.3 \\
\hline & IV & 100.1 & 133.6 & 74.9 \\
\hline \multirow[t]{5}{*}{2004} & I & 97.5 & 130.5 & 74.7 \\
\hline & II & 98.8 & 132.5 & 74.6 \\
\hline & III & 97.5 & 131.7 & 74.0 \\
\hline & IV & 94.7 & 128.2 & 73.9 \\
\hline & \multicolumn{4}{|c|}{ (Annual averages) } \\
\hline 2000 & & 108.8 & 142.0 & 76.6 \\
\hline 2001 & & 113.0 & 148.5 & 76.1 \\
\hline 2002 & & 109.9 & 146.7 & 74.9 \\
\hline 2003 & & 102.2 & 137.3 & 74.4 \\
\hline 2004 & & 97.1 & 130.7 & 74.3 \\
\hline
\end{tabular}

Source: Information Notice System, IMF.

1/ An increase in the index denotes an appreciation. 
Table 31. Dominica: External Public and Publicly Guaranteed Debt by Creditor, 2000-2004 1/

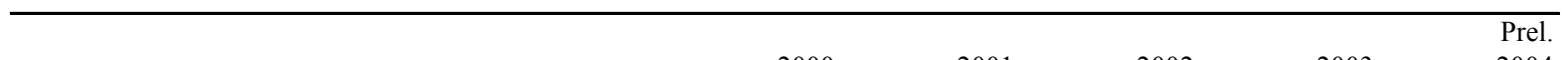

2000

2001

2002

2003

2004

(In millions of U.S. dollars)

Total debt outstanding, end of period

Bilateral debt

Agence Francaise de Developpement

Government of Trinidad and Tobago

Government of United Kingdom 2/

Investment Fund of Venezuela

Export-import Bank of Taiwan Province of China

Kuwait Fund for Arab Economic Development

Other bilaterals $3 /$

Multilateral debt

Caribbean Development Bank

European Development Bank

International Bank for Reconstruction and Development

International Development Association

International Fund for Agricultural Development

International Monetary Fund

Organization of Petroleum Exporting Countries

\section{Other debt}

Commercial banks

Other private

Arrears on interest payments 4/5/

Loan disbursements 6/

Debt service payments 6/

Principal repayments

Interest payments

Debt cancelation 7/

Change in interest arrears 5/

Valuation adjustment

Memorandum items:

External debt

External debt excluding interest arrears

External public debt service on a cash basis

\begin{tabular}{r}
$\mathbf{1 5 2 . 4}$ \\
$\mathbf{2 5 . 5}$ \\
12.8 \\
0.0 \\
0.0 \\
1.3 \\
8.5 \\
2.8 \\
0.0 \\
\\
$\mathbf{7 3 . 8}$ \\
51.4 \\
3.4 \\
2.4 \\
13.4 \\
2.8 \\
0.0 \\
0.4 \\
\hline \\
$\mathbf{5 3 . 1}$ \\
47.4 \\
5.1 \\
0.6
\end{tabular}

152.4

177.1

202.6

221.1

235.4

$\begin{array}{ll}25.5 & 37.3\end{array}$

37.3
12.1

0.0

9.3

1.3

11.0

3.7

0.0

84.2

60.4

4.1

2.7

14.0

2.9

0.0

0.2

55.5

49.9

5.1

0.5

27.7

27.1

$\mathbf{1 0 . 7}$

4.5

6.2

12.4

4.7

7.8

50.5

$\mathbf{5 0 . 5}$
12.0

52.6

$\mathbf{5 2 . 6}$
11.8

3.7

3.7

11.2

10.9

1.2

11.9

3.5

7.0

1.1

12.0

3.6

9.5

\section{9}

$\mathbf{1 1 0 . 4}$

$\begin{array}{ll}65.0 & 71.7\end{array}$

71.7
5.4

4.8

3.6

16.5

3.2

2.7

0.1

4.1

18.1

3.3

7.8

0.0

$\mathbf{5 6 . 2} \quad \mathbf{5 8 . 1}$

49.9

5.1

49.9

5.1

3.0

27.2

18.3

12.9

13.5

5.9

7.6

8.0

$-2.5$

0.0

0.0

$-0.4$

$-0.1$

0.0

0.0

1.9

4.2

2.5

4.2

(As a percentage of GDP)

56.6
56.4
4.0

67.6
67.5
4.7
85.6

84.4

5.2
86.9

85.2

5.2

Sources: Ministry of Finance, and Fund staff estimates.

$1 /$ The data shown in this table do not incorporate any changes resulting from the government's debt restructuring program.

2/ Loan provided by Societe Generale and guaranteed by the U.K. Government. The guarantee was exercised.

3/ Includes The Bahamas, Barbados, Belize, Grenada, and St. Vincent and the Grenadines.

4/ Principal arrears included in the debt stock.

5/ Includes arreas in dispute. Excludes arrears with Intercommercial Bank as debt with it was determined not to be the government's responsibility.

6/ On a cash basis.

7/ Refers to the agreement with Intercommercial Bank, which was determined not to be the government's responsibility. 